\title{
MODULI SPACES OF FRAMED SHEAVES ON CERTAIN RULED SURFACES OVER ELLIPTIC CURVES
}

\author{
THOMAS A. NEVINS
}

\begin{abstract}
Fix a ruled surface $S$ obtained as the projective completion of a line bundle $L$ on a complex elliptic curve $C$; we study the moduli problem of parametrizing certain pairs consisting of a sheaf $\mathcal{E}$ on $S$ and a map of $\mathcal{E}$ to a fixed reference sheaf on $S$. We prove that the full moduli stack for this problem is representable by a scheme in some cases. Moreover, the moduli stack admits an action by the group $\mathbf{C}^{*}$, and we determine its fixed-point set, which leads to explicit formulas for the rational homology of the moduli space.
\end{abstract}

\section{INTRODUCTION}

Moduli spaces of "sheaves with additional structure" of various sorts have been studied and used in many settings (see, for example, [Bra91], [Tha94], [KM99]), and there is a good general theory of moduli spaces for stable or semistable framed sheaves on complex projective surfaces thanks to work of Huybrechts-Lehn ([HL95b], [HL95a]). Moduli stacks of framed sheaves, however, are more natural objects of study than are the corresponding moduli spaces in some settings. For example, in the study of the restriction map

$$
\operatorname{Bun}_{S} \longrightarrow \operatorname{Bun}_{D}
$$

from the moduli stack of vector bundles on a variety $S$ to the moduli stack of vector bundles on a divisor $D$ in $S$, the stack-theoretic fibers turn out to be exactly moduli stacks of vector bundles on $S$ framed along $D$, and thus a natural first step in the study of such a restriction map is the study of the corresponding moduli stacks of framed bundles. Furthermore, in recent applications of algebraic geometry to questions in representation theory and noncommutative geometry ([Nak94b], [KKO00]) it is the full moduli stacks of framed sheaves that have been the natural objects of study.

In this paper we make a detailed study of the topology of one collection of moduli stacks of framed torsion-free sheaves on complex projective surfaces. Fix a complex elliptic curve $C$ and a line bundle $L$ of degree 0 on $C$. Let $S=\mathbf{P}(L \oplus \mathcal{O})$ denote the projectivization of the total space of the line bundle $L$; the surface $S$ is constructed from the total space of $L$ by adding a curve consisting of points at infinity in the fibers of $L$, and we denote this "curve at infinity" by $D$. Fix also a vector bundle $L_{1} \oplus L_{2}$ of rank two on $D$. We examine the moduli stack $\mathcal{M}_{S}\left(L_{1} \oplus L_{2}\right)$ parametrizing pairs $(\mathcal{E}, \phi)$ consisting of

1. a rank two torsion-free sheaf $\mathcal{E}$ on $S$, and

2. an isomorphism $\phi:\left.\mathcal{E}\right|_{D} \cong L_{1} \oplus L_{2}$.

1991 Mathematics Subject Classification. Primary 14D20; Secondary 14F25. 
Observe that $\mathbf{C}^{*}$ acts on $S$, thereby inducing an action on $\mathcal{M}_{S}\left(L_{1} \oplus L_{2}\right)$, and that there is a further action of $\left(\mathbf{C}^{*}\right)^{2}$ induced by scaling in the factors of $L_{1} \oplus L_{2}$. Of course, this stack is defined for a curve $C$ of any genus $g$ (and indeed a line bundle $L$ of any degree on $C$, although the stack is most interesting when $\operatorname{deg} L=2 g-2$ ). The case in which $g=0$ is already well understood (see [Nak94b], [Nak98]) through work of Nakajima; accordingly, the present work describes the stack and its topology in the first unknown case.

Theorem 5.3. Suppose that $C$ is an elliptic curve, that $L_{1}$ and $L_{2}$ are line bundles on $C$ both of the same arbitrary degree, and that $L$ is a line bundle of degree zero on $C$. Suppose further that $L, L_{1}$, and $L_{2}$ satisfy the very general conditions:

1. $L^{k}$ is nontrivial for all $k \in \mathbf{Z} \backslash\{0\}$; and

2. $L^{k} \otimes\left(L_{1}^{-1} \otimes L_{2}\right)$ is nontrivial for all $k \in \mathbf{Z}$.

Then if $S=\mathbf{P}(L \oplus \mathcal{O})$, the moduli stack $\mathcal{M}_{S}\left(L_{1} \oplus L_{2}\right)$ is a smooth separated scheme. Moreover $\mathcal{M}_{S}\left(L_{1} \oplus L_{2}\right)$ is exhausted by open subschemes that are equivariantly quasiprojective with respect to the natural action of $\left(\mathbf{C}^{*}\right)^{3}$ on $\mathcal{M}_{S}\left(L_{1} \oplus L_{2}\right)$.

In Section 6 we go on to give an explicit homology basis for $\mathcal{M}_{S}\left(L_{1} \oplus L_{2}\right.$ ) (see Theorem 6.1). One may stratify $\mathcal{M}_{S}\left(L_{1} \oplus L_{2}\right)$ as

$$
\mathcal{M}_{S}\left(L_{1} \oplus L_{2}\right)=\coprod \mathcal{M}_{S}\left(L_{1} \oplus L_{2}\right)\left(c_{1}, c_{2}, l\right)
$$

where $c_{1}=-l^{\prime} \sigma$ and $c_{2}$ are the Chern classes of the sheaf $\mathcal{E}$ and $l$ describes the "generic splitting type" of $\mathcal{E}$ (see Sections 3 and 6 for more information). From Theorem 6.1 , the homology of $\mathcal{M}_{S}\left(L_{1} \oplus L_{2}\right)$ is a direct sum of homology groups $H_{k}\left(l^{\prime}, c_{2}, l\right)$ "living on" the strata $\mathcal{M}_{S}\left(L_{1} \oplus L_{2}\right)\left(c_{1}, c_{2}, l\right)$ from (1.2) and, using this decomposition, the homology assembles into a generating function:

$$
\begin{gathered}
\sum_{l^{\prime}, c_{2}, l, k} \operatorname{dim}\left(H_{k}\left(l^{\prime}, c_{2}, l\right)\right) x^{k} u^{l^{\prime}} v^{c_{2}} w^{l}= \\
{\left[\sum_{l}\left(\prod_{j \geq 1} \frac{\left(1+x^{4 j-3} v^{j}\right)^{2}}{\left(1-x^{4 j-4} v^{j}\right)\left(1-x^{4 j-2} v^{j}\right)}\right) u^{2 l} w^{-l}\right]+} \\
{\left[\sum_{l, l^{\prime}}\left(\prod_{j>l^{\prime}+2 l} \frac{\left(1+x^{4 j-3} v^{j}\right)^{2}}{\left(1-x^{4 j-4} v^{j}\right)\left(1-x^{4 j-2} v^{j}\right)}\right)\left(\prod_{1 \leq j \leq l^{\prime}+2 l} \frac{\left(1+x^{4 j-1} v^{j}\right)}{\left(1-x^{4 j-2} v^{j}\right)\left(1-x^{4 j} v^{j}\right)}\right) \times\right.} \\
\times \\
\times\left(\frac{\left(1+x^{4\left(l^{\prime}+2 l\right)-3} v^{l^{\prime}+2 l}\right)^{2}}{\left(1-x^{4\left(l^{\prime}+2 l\right)-4} v^{l^{\prime}+2 l}\right)\left(1-x^{4\left(l^{\prime}+2 l\right)-2} v^{l^{\prime}+2 l}\right)} u^{l^{\prime}} w^{l}+\right. \\
\left.\left.\frac{\left(1+x^{4\left(l^{\prime}+2 l\right)-1} v^{l^{\prime}+2 l}\right)^{2}}{\left(1-x^{4\left(l^{\prime}+2 l\right)-2} v^{l^{\prime}+2 l}\right)\left(1-x^{4\left(l^{\prime}+2 l\right)-2} v^{l^{\prime}+2 l}\right)} u^{-l^{\prime}} w^{-l}\right)\right] .
\end{gathered}
$$

Although the techniques used here to obtain the homology formulas are standard, it is only the rather unexpected conjunction of the results of Sections 4 and 5 that makes possible the application of known techniques to compute the homology (and the generating function) in Section 6. Indeed, as we have already mentioned the stack $\mathcal{M}_{S}\left(L_{1} \oplus L_{2}\right)$ has a $\mathbf{C}^{*}$ action coming from the action on $S$ as well as a $\left(\mathbf{C}^{*}\right)^{2}$ action by scaling in the factors of $L_{1} \oplus L_{2}$, and one would like to use this action to produce a homology basis for the stack. In order to apply the usual 
localization theorems, however, one generally needs to be working with either a smooth projective algebraic manifold or a smooth Hausdorff Kähler manifold for which limits $\lim _{\lambda \rightarrow 0} \lambda \cdot m$ exist for all points $m$ of the manifold (here $\lambda \in \mathbf{C}^{*}$ ). It turns out that, although the stack $\mathcal{M}_{S}\left(L_{1} \oplus L_{2}\right)$ is not even of finite type, Theorem 5.3, in conjunction with the results of Section 4, provides just enough leverage to allow the application of localization formulas in their usual form. The methods used to prove Theorem 5.3 seem to be new, as do the applications of Langton's ([Lan75]) ideas in Section 4.

While one is inclined to believe that, in general, moduli stacks are more cumbersome and strange than the associated coarse moduli spaces, a remarkable story is beginning to emerge that suggests that the moduli stacks of framed sheaves on complex projective surfaces often behave quite reasonably (see also [Nev00b], [Nev00a] for progress in this direction), and thus that a detailed study of the geometry of these stacks might not be an unreasonable goal. Taken in this light, the present paper both illuminates the kind of behavior one might reasonably expect to see from these stacks in general and develops a picture of the topology in some special cases, cases which, moreover, seem to have the potential for some striking applications.

One potential application of the story developed in this paper is to the geometric Langlands program for surfaces. Given a curve $C$ in a surface $S$, one wants to compute the algebra of Hecke operators arising from modification of sheaves on $S$ along the curve $C$. If one allows only point modifications along $C$, then one knows the algebra completely, thanks to work of Nakajima ([Nak97]), Grojnowski ([Gro96]) and Baranovsky ([Bar]). If, however, one allows modification along the entire curve $C$, the computation of the algebra appears to be extremely difficult, although progress in a special case (one which generalizes the special case of our setting in which the curve $C$ is the projective line and the line bundle $L$ is $\mathcal{O}(-2))$ by Nakajima ([Nak94b], [Nak98]) and Ginzburg-Kapranov-Vasserot ([GKV95], [KV00]) suggests that the picture should be a very rich one. It seems that the appropriate algebra is unknown even when the curve is an elliptic curve - but see [GKV95] for a very interesting conjecture - and thus the topological picture developed in this paper might provide one input for the computation of a completely new class of geometrically constructed representations of algebras.

Another direction for further investigation concerns the restriction morphism (1.1). When $S$ is a K3 surface and $D$ is an elliptic curve, this morphism of stacks is expected to have remarkable geometric properties, and it seems plausible that the information obtained in this paper may provide a starting place for exploring that morphism in detail.

The author is deeply indebted to his dissertation advisor, Kevin Corlette, without whose help, guidance and encouragement this work could never have been completed. The author is also grateful to V. Baranovsky, S. Chang, V. Ginzburg, B. Hassett, R. Lazarsfeld, M. Mandell, R. Narasimhan, S. Nevins, M. Nori, T. Pantev, A. Prasad, and I. Robertson for helpful conversations. The author's graduate work, of which this paper is a result, was supported in part by an NDSEG fellowship from the Office of Naval Research. 


\section{The Moduli Stack: Definition and Group Action}

In this section, we define the moduli stacks in which we are interested and describe some relevant properties of the group action.

2.1. Notation and Definition of the Moduli Stack. Fix a smooth complete irreducible complex curve $C$, a line bundle $L$ on $C$, and a rank two vector bundle $E$ on $C$; later we will specialize to the case in which $C$ has genus one and $E$ splits as $E=L_{1} \oplus L_{2}$. Let

$$
S=\mathbf{P}(L \oplus \mathcal{O}),
$$

and let $D$ denote the divisor at infinity in $S$. We will use the notation $\sigma$ for the curve $C$ embedded in $S$ as the zero section of the bundle $L$. Since $D \cong C$ canonically via the projection map $\pi: S \rightarrow C$, we may think of $E$ as a vector bundle on $D$ or on $\sigma$. If $R$ is a $\mathbf{C}$-scheme, let $E_{R}$ denote the pullback of $E$ to $D_{R}=D \times R$.

Definition 2.1. Let $\mathcal{M}_{S}(E)$ denote the moduli stack of $E$-framed rank two torsionfree sheaves on $S$; this stack has as its objects pairs $\left(\mathcal{E}_{R}, \phi_{R}\right)$ consisting of an $R$-flat family of rank two torsion-free sheaves $\mathcal{E}_{R}$ on $S_{R}$ together with an isomorphism

$$
\phi_{R}:\left.\mathcal{E}_{R}\right|_{D_{R}} \stackrel{\sim}{\longrightarrow} E_{R} .
$$

A morphism $\left(\mathcal{E}_{R}, \phi_{R}\right) \rightarrow\left(\mathcal{E}_{T}^{\prime}, \phi_{T}^{\prime}\right)$ consists of a morphism $f: R \rightarrow T$ together with an isomorphism

$$
\mathcal{E}_{R} \stackrel{\psi}{\sim}\left(1_{S} \times f\right)^{*} \mathcal{E}_{T}^{\prime}
$$

for which

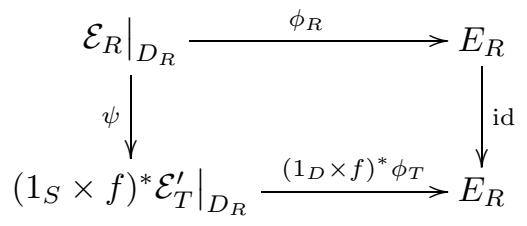

commutes.

Proposition 2.2. ([Nev00a]) $\mathcal{M}_{S}(E)$ is an algebraic stack that is locally of finite type over Spec C.

Sketch of Proof. Let $\mathrm{TF}_{S}$ denote the moduli stack parametrizing torsion-free sheaves on $S$, and let $\mathrm{TF}_{S}(D)$ denote its open substack that parametrizes those sheaves that are locally free along $D$ (see [LMB92]). There is a restriction morphism

$$
\mathrm{TF}_{S}(D) \rightarrow \operatorname{Bun}(D),
$$

where $\operatorname{Bun}(D)$ denotes the moduli stack parametrizing vector bundles on $D$. The vector bundle $E$ on $D$ determines a morphism $\operatorname{Spec} \mathbf{C} \rightarrow \operatorname{Bun}(D)$, and $\mathcal{M}_{S}(E)$ is the fiber product of the diagram

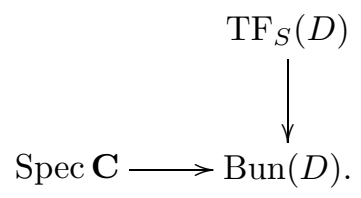

The proposition follows because $\operatorname{Spec} \mathbf{C} \rightarrow \operatorname{Bun}(D)$ is an $\operatorname{Aut}(E)$-torsor over a locally closed substack of $\operatorname{Bun}(D)$. 
2.2. The Group Action on the Surface $S$. Let $L$ denote a line bundle on the curve $C$. Let $\mathcal{O}$ denote the trivial bundle on $C$. Let $S=\operatorname{Proj} \operatorname{Sym}^{\bullet}\left(L^{*} \oplus \mathcal{O}^{*}\right)$. An action of $\mathbf{C}^{*}$ on $\mathbf{P}^{1}$ is given by a map $\mathbf{C}^{*} \times \mathbf{P}^{1} \stackrel{m}{\longrightarrow} \mathbf{P}^{1}$ and hence is determined by the map of rings

$$
\mathcal{O}_{\mathbf{P}^{1}} \longrightarrow m_{*} \mathcal{O}_{\mathbf{C}^{*} \times \mathbf{P}^{1}}=\mathcal{O}_{\mathbf{P}^{1}}\left[\lambda, \lambda^{-1}\right] .
$$

Globally on $S$, then, to define an action of $\mathbf{C}^{*}$ on $S$ we need a map

$$
\operatorname{Sym}^{\bullet}\left(L^{*} \oplus \mathcal{O}^{*}\right) \stackrel{\bar{m}}{\longrightarrow} \operatorname{Sym}^{\bullet}\left(L^{*} \oplus \mathcal{O}^{*}\right)\left[\lambda, \lambda^{-1}\right] ;
$$

to obtain the usual action on $\mathbf{P}^{1}$ as the appropriate specialization at a fixed $\lambda \in \mathbf{C}^{*}$ in each fiber, we must take $L^{*} \mapsto L^{*}$ and $\mathcal{O}^{*} \mapsto \lambda^{-1} \mathcal{O}^{*}$.

Remark 2.3. In our applications to $\mathcal{M}_{S}(E)$, we will want to pull sheaves back along the inverse action, i.e., the action by which $\lambda \in \mathbf{C}^{*}$ acts on $S$ via the usual action of $\lambda^{-1}$ on $S$. It is convenient in our discussion to compute everything for the standard action on $S$ and then later to simply reverse the weights of the $\mathbf{C}^{*}$ action when we are concerned with the inverse action.

Choose local generators $l^{*}$ of $L^{*}$ and $s^{*}$ of $\mathcal{O}^{*}$. We will translate between $\operatorname{Sym}^{\bullet}\left(L^{*} \oplus \mathcal{O}^{*}\right)$ and $\mathbf{C}\left[l^{*}, s^{*}\right]$ and thus describe some of the usual structures on $S$.

The zero section $\sigma$ of $L \subset S$ is just the set along which the section $l^{*}$ vanishes, hence $\mathcal{O}_{\sigma}=\operatorname{Sym}^{\bullet}\left(L^{*} \oplus \mathcal{O}^{*}\right) /\left\langle L^{*}\right\rangle$, where $\left\langle L^{*}\right\rangle$ denotes the ideal generated by $L^{*}$. Now $\left\langle L^{*}\right\rangle$ is exactly the image of $L^{*} \otimes \operatorname{Sym}^{\bullet}\left(L^{*} \oplus \mathcal{O}^{*}\right)$ in $\operatorname{Sym}^{\bullet}\left(L^{*} \oplus \mathcal{O}^{*}\right)$ under multiplication, so

$$
\mathcal{O}(-\sigma)=\left[L^{*}(-1) \otimes \operatorname{Sym}^{\bullet}\left(L^{*} \oplus \mathcal{O}^{*}\right)\right]^{\sim} .
$$

Here the notation $L^{*}(-1)$ indicates that the grading on $L^{*}$ is shifted so that it now lies in graded degree 1 ; this makes the inclusion

$$
L^{*}(-1) \otimes \operatorname{Sym}^{\bullet}\left(L^{*} \oplus \mathcal{O}^{*}\right) \subset \operatorname{Sym}^{\bullet}\left(L^{*} \oplus \mathcal{O}^{*}\right)
$$

compatible with the gradings and consequently gives a map of the associated coherent sheaves (indicated by the tilde) on $\operatorname{Proj} \operatorname{Sym}^{\bullet}\left(L^{*} \oplus \mathcal{O}^{*}\right)$.

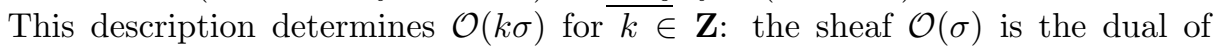
$\mathcal{O}(-\sigma)$ or $\mathcal{O}(\sigma)=\left[L(1) \otimes \operatorname{Sym}^{\bullet}\left(L^{*} \oplus \mathcal{O}^{*}\right)\right]^{\sim}$, and $\mathcal{O}(k \sigma)$ is therefore $\mathcal{O}(k \sigma)=$ $\left[L^{k}(k) \otimes \operatorname{Sym}^{\bullet}\left(L^{*} \oplus \mathcal{O}^{*}\right)\right]^{\sim}$. In terms of the coordinate ring $\mathbf{C}\left[l^{*}, s^{*}\right]$ we get $\mathcal{O}(k \sigma)=$ $\left(l^{*}\right)^{-k} \mathbf{C}\left[l^{*}, s^{*}\right]^{\sim}$, where here $\left(l^{*}\right)^{-k}$ lies, as expected, in graded degree $-k$.

A structure of $\mathbf{C}^{*}$-equivariant $\mathcal{O}_{S^{-}}$-module on $\widetilde{M}$ is given by an isomorphism $m^{*} M \stackrel{I}{\rightarrow} M\left[\lambda, \lambda^{-1}\right]$ of $\operatorname{Sym}^{\bullet}\left(L^{*} \oplus \mathcal{O}^{*}\right)\left[\lambda, \lambda^{-1}\right]$-modules with certain compatibility conditions (see [Vie95]). The action of an element $\lambda \in \mathbf{C}^{*}$ on sections of $\widetilde{M}$ is then given by the composition

$$
M \stackrel{m^{*}}{\longrightarrow} m^{*} M \stackrel{I}{\longrightarrow} M\left[\lambda, \lambda^{-1}\right] \stackrel{\operatorname{eval}(\lambda)}{\longrightarrow} M .
$$

We want to use the equivariant structures on submodules of $\mathcal{O}_{S}$ induced from the canonical equivariant structure on $\mathcal{O}_{S}$ that comes from the group action on $S$.

Over $S \backslash D$, the sheaf $\mathcal{O}(k \sigma)$ consists of elements of $\left(l^{*}\right)^{-k} \mathbf{C}\left[l^{*}, s^{*}\right]_{s^{*}}$ of degree zero; this module has generator $\left(\frac{s^{*}}{l^{*}}\right)^{k}$, on which $\mathbf{C}^{*}$ acts with weight $-k$ owing to the $\left(s^{*}\right)^{k}$ term in the numerator: under the map $\mathcal{O}_{S} \longrightarrow \mathcal{O}_{S}\left[\lambda, \lambda^{-1}\right]$, the generator 
$\left(\frac{s^{*}}{l^{*}}\right)^{k}$ of $\mathcal{O}(k \sigma)($ for $k \leq 0)$ maps to $\lambda^{-k}\left(\frac{s^{*}}{l^{*}}\right)^{k}$, so this element lies in the weight $-k$ subspace.

Remark 2.4. Note, again, that this implies that the generator lies in the weight $k$ subspace for the inverse action. We will summarize our results for the inverse action in Proposition 2.6.

Over $S \backslash \sigma$ we find that $\mathcal{O}(k \sigma)$ consists of elements of $\left(l^{*}\right)^{-k} \mathbf{C}\left[l^{*}, s^{*}\right] l^{*}$ of degree zero, and this module has as its generator the unit of the ring $\mathbf{C}\left[s^{*} / l^{*}\right]$, on which $\mathbf{C}^{*}$ acts with trivial weight. Thus, $\mathbf{C}^{*}$ acts with weight zero in the fibers of $\mathcal{O}(k \sigma)$ along $D$ but with weight $-k$ in the fibers of $\mathcal{O}(k \sigma)$ along $\sigma$.

It is convenient here to note also that, since the ring $\mathcal{O}_{S}$ on $S \backslash \sigma$ comes from $\mathbf{C}\left[s^{*} / l^{*}\right]$, the group $\mathbf{C}^{*}$ acts on functions defined near $D$ as follows: if $f$ is a function homogeneous in the variable $s^{*} / l^{*}$, then $\mathbf{C}^{*}$ acts on $f$ with weight equal to minus the order of vanishing of $f$ along $D$. Similarly, near $\sigma$ the group $\mathbf{C}^{*}$ acts with weight $k$ on the functions homogeneous in the variable $l^{*} / s^{*}$ vanishing to order exactly $k$ along $\sigma$.

Recall that the space of global sections of a module $\mathcal{O}(n)=\widetilde{\Gamma(\mathcal{O}(n)})$ over $\mathbf{P}^{1}$ is just the space of degree zero elements of the graded module $\Gamma_{*}(\mathcal{O}(n)$ ) (see [Har77] for this notation). The construction of our line bundles allows us to conclude that the modules by means of which we defined these line bundles are already in the image of the functor $\Gamma_{*}$, and hence, if $\pi: S \rightarrow C$ is the canonical projection, that $\pi_{*} \mathcal{O}(k \sigma)=\left(L^{k} \otimes \operatorname{Sym}^{k}\left(L^{*} \oplus \mathcal{O}^{*}\right)\right)$. Of course if $k<0$ this is the zero sheaf; however, if $k \geq 0$ one obtains

$$
\pi_{*} \mathcal{O}(k \sigma)=\sum_{j=0}^{k} L^{k} \otimes\left(L^{*}\right)^{j} \otimes\left(\mathcal{O}^{*}\right)^{k-j}=\sum_{j=0}^{k} L^{k-j} \otimes\left(\mathcal{O}^{*}\right)^{k-j}
$$

If we identify this direct sum with $\pi_{*} \mathcal{O}(k \sigma)=\sum_{j=0}^{k} L^{k-j}$, then our earlier description implies that $\mathbf{C}^{*}$ acts on the factor $L^{k-j}$ with weight $j-k$.

We want to include one further, similar calculation. Performing the same analysis as above for

$$
\mathcal{O}(-D)=\left\langle\mathcal{O}^{*}\right\rangle \subset \operatorname{Sym}^{\bullet}\left(L^{*} \oplus \mathcal{O}^{*}\right)
$$

one gets

$$
\mathcal{O}(-k D)=\left(\mathcal{O}^{*}\right)^{k}(-k) \otimes \operatorname{Sym}^{\bullet}\left(L^{*} \oplus \mathcal{O}^{*}\right) .
$$

In terms of the ring $\mathbf{C}\left[l^{*}, s^{*}\right]$, one then obtains $\left(s^{*}\right)^{k}(-k) \mathbf{C}\left[l^{*}, s^{*}\right]$, and one may describe this sheaf near $D$ as the sheaf associated to the module of degree zero elements of $\left(s^{*}\right)^{k} \mathbf{C}\left[l^{*}, s^{*} l_{l^{*}}\right.$. This module has generator $\left(s^{*} / l^{*}\right)^{k}$ and so $\mathbf{C}^{*}$ acts on its generator with weight $-k$.

Remark 2.5. The description in this section of weights of elements in various sheaves easily allows computation of weights in, for example, $\mathbf{C}^{*}$-invariant finite-colength ideal sheaves or their quotients as well, simply because these weights are induced from those in the structure sheaf of $S$.

We now summarize our conclusions, but for the inverse action.

Proposition 2.6. For the inverse action $m^{-1}(\lambda, s)=\lambda^{-1} \cdot s$ of $\mathbf{C}^{*}$ on $s \in S$ and the induced equivariant structures on sheaves coming from $\mathcal{O}_{S}$, 
1. the homogeneous generator of $\mathcal{O}(k \sigma)$ along $\sigma$ lies in weight $k$, and the homogeneous generator along $D$ lies in weight zero;

2. $\pi_{*} \mathcal{O}(k \sigma)=\sum_{j=0}^{k} L^{k-j}$

and $\mathbf{C}^{*}$ acts on $L^{k-j}$ with weight $k-j$;

3. $\mathbf{C}^{*}$ acts on $I_{D}^{k} / I_{D}^{k+1} \cong L^{k}$ with weight $k$; and

4. $\mathbf{C}^{*}$ acts on the homogeneous functions that vanish to order $k$ along $\sigma$ with weight $-k$.

\subsection{The Group Action on $\mathcal{M}_{S}(E)$.}

Construction 2.7. The stack $\mathcal{M}_{S}(E)$ admits a $\mathbf{C}^{*}$ action as follows: $\lambda \in \mathbf{C}^{*}$ acts by pulling back along the map $m_{\lambda^{-1}}: S \rightarrow S$ given by multiplication by $\lambda^{-1}$ on $S$ fiberwise, which extends the action on the total space of the line bundle $L$ by scaling in the fibers.

Suppose that

$$
0 \rightarrow M_{1} \rightarrow M_{2} \rightarrow M_{3} \rightarrow 0
$$

is a short exact sequence of $\mathcal{O}_{S}$-modules and that $M_{1}$ and $M_{3}$ are equipped with $\mathbf{C}^{*}$ equivariant structures. Suppose $M_{2}$ is defined by a Čech 1-cocycle $\theta \in Z^{1}\left(S, \underline{\operatorname{Hom}}\left(M_{3}, M_{1}\right)\right)$. Then $m_{\lambda^{-1}}^{*} M_{2}$ occurs as a 1-extension

$$
0 \rightarrow m_{\lambda^{-1}}^{*} M_{1} \rightarrow m_{\lambda^{-1}}^{*} M_{2} \rightarrow m_{\lambda^{-1}}^{*} M_{3} \rightarrow 0
$$

and, if

$$
I_{1}: m_{\lambda^{-1}}^{*} M_{1} \longrightarrow M_{1} \quad \text { and } \quad I_{3}: m_{\lambda^{-1}}^{*} M_{3} \longrightarrow M_{3}
$$

are the isomorphisms coming from the $\mathbf{C}^{*}$-equivariant structure, then $m_{\lambda^{-1}}^{*} \theta$ yields the 1-cocycle

$$
I_{1} \circ m_{\lambda^{-1}}^{*} \theta \circ I_{3}^{-1} \in Z^{1}\left(S, \underline{\operatorname{Hom}}\left(M_{3}, M_{1}\right)\right)
$$

that determines $m_{\lambda^{-1}}^{*} M_{2}$ as an extension of $M_{3}$ by $M_{1}$. Hence $m_{\lambda^{-1}}^{*} M_{2}$ is determined by the class

$$
\left[\lambda^{-1} \cdot \theta\right] \in H^{1}\left(S, \underline{\operatorname{Hom}}\left(M_{3}, M_{1}\right)\right) .
$$

In order to determine the invariant 1-extensions, then, we need only determine the invariants in such cohomology (or, more generally, Ext) groups. The machinery of the next section makes it possible to use this data to determine fixed points of $\mathcal{M}_{S}(E)$.

\section{The Fixed-Point Set}

We begin by describing a technical device that allows concrete study of sheaves on $S$ by means of extensions.

3.1. The Canonical Short Exact Sequence. Let $S=\mathbf{P}(L \oplus \mathcal{O}), L$ a line bundle on the curve $C$. We will describe the canonical short exact sequence associated to a rank two torsion-free sheaf on $S$. 
3.1.1. The Short Exact Sequence for Sheaves of Type U. We first consider the case of a rank two torsion-free sheaf on $S$ of unequal generic splitting type, or type $U$; by this we mean a sheaf $\mathcal{E}$ whose restriction to a generic fiber $f$ of $S \rightarrow C$ is of the form

$$
\left.\mathcal{E}\right|_{f} \cong \mathcal{O}\left(d_{1}\right) \oplus \mathcal{O}\left(d_{2}\right)
$$

with $d_{1} \neq d_{2}$. Suppose $\mathcal{E}$ is such a sheaf that is in addition locally free, and suppose $d_{1}>d_{2}$. The canonical short exact sequence associated to $\mathcal{E}$ is the sequence

$$
0 \rightarrow\left[\pi^{*}\left(\pi_{*} \mathcal{E}\left(-d_{1} \sigma\right)\right)\right]\left(d_{1} \sigma\right) \rightarrow \mathcal{E} \rightarrow Q \rightarrow 0
$$

This exact sequence has been used widely to study sheaves on rational and ruled surfaces; we will use the papers of Brosius ([Bro83a], [Bro83b]) as our canonical references for this sequence.

Proposition 3.1. (Brosius) If $\mathcal{E}$ is locally free of type $U$, then $Q$ is a rank one torsion-free sheaf on $S$; hence the exact sequence is a 1-extension of the form

$$
0 \rightarrow L_{1} \rightarrow \mathcal{E} \rightarrow L_{2} \otimes I_{2} \rightarrow 0
$$

where $L_{1}$ and $L_{2}$ are line bundles on $S$ and $I_{2}$ is an ideal of finite colength. If $\mathcal{E}_{T}$ is a $T$-flat family of rank two vector bundles on $S$ where $T$ is some integral Noetherian base scheme and the generic splitting type $\left.\mathcal{E}_{t}\right|_{f} \cong \mathcal{O}\left(d_{1}\right) \oplus \mathcal{O}\left(d_{2}\right)$ is constant as $t \in T$ varies, then the canonical subsheaf $\left(L_{1}\right)_{T}$ for the family specializes properly: $\left(\left(L_{1}\right)_{T}\right)_{t}=\left(L_{1}\right)_{t}$.

There is a similar short exact sequence of the form

$$
0 \rightarrow L_{1} \otimes I_{1} \rightarrow \mathcal{E} \rightarrow L_{2} \otimes I_{2} \rightarrow 0
$$

when $\mathcal{E}$ fails to be locally free; here $L_{1}$ and $L_{2}$ are line bundles and $I_{1}$ and $I_{2}$ are finite colength ideals. This short exact sequence has two useful descriptions.

1. Given $\mathcal{E}$ torsion-free of generic splitting type $\left.\mathcal{E}\right|_{f}=\mathcal{O}\left(d_{1}\right) \oplus \mathcal{O}\left(d_{2}\right)$, let

$$
\mathcal{L}:=\left[\pi^{*}\left(\pi_{*} \mathcal{E}\left(-d_{1} \sigma\right)\right)\right]\left(d_{1} \sigma\right) .
$$

Then $L_{1} \otimes I_{1} \subset \mathcal{E}$ is the saturation of $\mathcal{L}$ in $\mathcal{E}$, and by definition the quotient $\mathcal{E} /\left(L_{1} \otimes I_{1}\right)$ is thus a torsion-free sheaf of rank one.

2. Apply the construction of Brosius to $\mathcal{E}^{* *}$; one gets a diagram

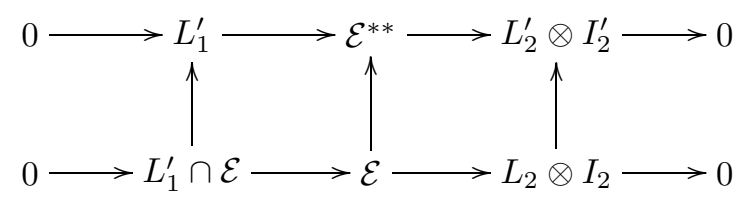

for which the vertical arrows are inclusions. Then $L_{1} \otimes I_{1}=L_{1}^{\prime} \cap \mathcal{E}$ is exactly as in description 1.

Remark 3.2. Notice that if $\mathcal{E}$ is $\mathbf{C}^{*}$-equivariant, then by construction both $\left(\pi^{*}\left[\pi_{*} \mathcal{E}\left(-d_{1} \sigma\right)\right]\right)\left(d_{1} \sigma\right)$ and (as then follows from the first description) $L_{1} \otimes I_{1}$ are $\mathbf{C}^{*}$-invariant subsheaves of $\mathcal{E}$.

One may show, using techniques similar to those employed by Brosius, that the canonical exact sequence specializes properly when $\mathcal{E}$ varies in families of torsionfree sheaves, provided one fixes the proper invariants; however, we will not need this fact. 
3.1.2. The Short Exact Sequence for Sheaves of Type E. Suppose now that $\mathcal{E}$ is of equal generic splitting type or type $E$ : this means that the restriction of $\mathcal{E}$ to the generic fiber $f$ is of the form $\left.\mathcal{E}\right|_{f} \cong \mathcal{O}(d) \oplus \mathcal{O}(d)$. We will assume that $\mathcal{E}$ is locally free, since this will suffice for our intended application later in the section. In this case, one obtains a canonical short exact sequence

$$
0 \rightarrow\left(\pi^{*} E^{\prime}\right) \otimes \mathcal{O}(d \sigma) \rightarrow \mathcal{E} \rightarrow \mathcal{I}_{Z \subset X} \otimes \mathcal{O}(d \sigma) \rightarrow 0
$$

where

$$
\left(\pi^{*} E^{\prime}\right) \otimes \mathcal{O}(d \sigma)=\pi^{*}\left(\pi_{*} \mathcal{E}(-d \sigma)\right)(d \sigma)
$$

and $\mathcal{I}_{Z \subset X}$ is the ideal associated to the inclusion of an l.c.i. (that is, local complete intersection) zero-cycle $Z \subset S$ in the scheme $X=\pi^{-1}(\pi(Z)) \subset S$. In fact, if $\pi^{*} E^{\prime}$ is the vector bundle above, then $Z$ is exactly the zero-cycle along which the map $\pi^{*} E^{\prime} \rightarrow \mathcal{E}(-d \sigma)$ of rank two vector bundles fails to be a rank two linear map of the fibers.

3.2. The Fixed Points for the Action. We proceed to study the fixed points of $\mathbf{C}^{*}$ in $\mathcal{M}_{S}(E)$. We begin by proving that any simple fixed point admits a $\mathbf{C}^{*}$ equivariant structure.

In what follows, let $m: \mathbf{C}^{*} \times S \rightarrow S$ denote the (inverse) multiplication map given by $m(\lambda, s)=\lambda^{-1} \cdot s$. We also let $m_{\lambda}$ denote the map $m(\lambda, \cdot): S \rightarrow S$ given by multiplication by $\lambda^{-1}$.

Lemma 3.3. Let $R$ denote any $\mathbf{C}$-scheme. Suppose $\mathcal{E}_{R}$ is an $R$-flat family of $R$ simple torsion-free rank two coherent sheaves on $S$ that is equipped with a framing $\left.\mathcal{E}_{R}\right|_{D_{R}} \stackrel{\phi_{R}}{\longrightarrow} E_{R}$. Suppose furthermore that for each $\lambda \in \mathbf{C}^{*}$, one has $m_{\lambda}^{*}\left(\mathcal{E}_{R}, \phi_{R}\right) \cong$ $\left(\mathcal{E}_{R}, \phi_{R}\right)$. Then $\mathcal{E}_{R}$ admits a $\mathbf{C}^{*}$-equivariant structure for which $\phi_{R}$ is $\mathbf{C}^{*}$-equivariant.

Proof. Let $p: \mathbf{C}^{*} \times S \rightarrow S$ denote the projection on the second factor, let $q: S \times R \rightarrow$ $R$ denote the projection to $R$, and, by abuse of notation, let $q: D \times R \rightarrow R$ also denote the projection to $R$.

By assumption, we have $m_{\lambda}^{*} \mathcal{E}_{R} \cong \mathcal{E}_{R}$ for each $\lambda \in \mathbf{C}^{*}$. Since $\mathcal{E}_{R}$ is $R$-simple, we find that $\operatorname{Ext}_{q \mid \lambda}^{0}\left(m_{\lambda}^{*} \mathcal{E}_{R},\left(p^{*} \mathcal{E}_{R}\right)_{\lambda}\right)$ is a line bundle on $R$ for each $\lambda \in \mathbf{C}^{*}$; therefore, we get a line bundle $\operatorname{Ext}_{(\mathrm{id} \times q)}^{0}\left(m^{*} \mathcal{E}_{R}, p^{*} \mathcal{E}_{R}\right)$ on $\mathbf{C}^{*} \times R$. There is a restriction map

since

$$
\operatorname{Ext}_{(\mathrm{id} \times q)}^{0}\left(m^{*} \mathcal{E}_{R}, p^{*} \mathcal{E}_{R}\right) \rightarrow \operatorname{Ext}_{(\mathrm{id} \times q)}^{0}\left(\left.m^{*} \mathcal{E}_{R}\right|_{D_{R}},\left.p^{*} \mathcal{E}_{R}\right|_{D_{R}}\right)
$$

$$
\left.m^{*} \mathcal{E}_{R}\right|_{D_{R}} \cong E_{\mathbf{C}^{*} \times R} \text { and }\left.p^{*} \mathcal{E}_{R}\right|_{D_{R}} \cong E_{\mathbf{C}^{*} \times R}
$$

canonically via $\phi_{R}$, we find that

$$
\begin{aligned}
\operatorname{Ext}_{(\mathrm{id} \times q)}^{0}\left(\left.m^{*} \mathcal{E}_{R}\right|_{D_{R}},\left.p^{*} \mathcal{E}_{R}\right|_{D_{R}}\right) & \cong \operatorname{Ext}_{(\mathrm{id} \times q)}^{0}\left(E_{\mathbf{C}^{*} \times R}, E_{\mathbf{C}^{*} \times R}\right) \\
& \cong \mathcal{O}_{\mathbf{C}^{*} \times R}
\end{aligned}
$$

canonically. Because each $m_{\lambda}^{*}\left(\mathcal{E}_{R}, \phi_{R}\right)$ is isomorphic to $\left(\mathcal{E}_{R}, \phi_{R}\right)$, the induced map of line bundles

$$
\operatorname{Ext}_{(\mathrm{id} \times q)}^{0}\left(m^{*} \mathcal{E}_{R}, p^{*} \mathcal{E}_{R}\right) \rightarrow \mathcal{O}_{\mathbf{C}^{*} \times R}
$$

is surjective and therefore is an isomorphism (see Theorem 2.4 of [Mat89]). The inverse image of the identity section $1 \in \mathcal{O}_{\mathbf{C}^{*} \times R}$ now gives a section of $\operatorname{Ext}_{(\mathrm{id} \times q)}^{0}\left(m^{*} \mathcal{E}_{R}, p^{*} \mathcal{E}_{R}\right)$; 
moreover, the unicity of our construction guarantees commutativity of the necessary diagram (see Section 3.2 of [Vie95]) to make the pulled-back morphism $m^{*} \mathcal{E}_{R} \rightarrow p^{*} \mathcal{E}_{R}$ an equivariant structure on $\mathcal{E}_{R}$. This completes the proof.

Remark 3.4. In the concrete cases in which we will be interested in Sections 5 and 6 , all $E$-framed sheaves that are invariant under each $\lambda \in \mathbf{C}^{*}$ will admit $\mathbf{C}^{*}$ equivariant structures. In fact, the proper notion of a $\mathbf{C}^{*}$-fixed point in our stack is a $\mathbf{C}^{*}$-equivariant framed sheaf in general; however, because we will not need this level of generality in this work, we will omit a detailed explanation of the reasons that this is the appropriate "fixed-point substack."

3.2.1. Fixed Points: The Unequal Case. Now suppose that $(\mathcal{E}, \phi)$ defines a $\mathbf{C}^{*}$ invariant point of $\mathcal{M}_{S}(E)(\operatorname{Spec} \mathbf{C})$ and that $\mathcal{E}$ is of type $U$. Replacing $\mathcal{E}$ by its double dual, we assume for the moment that $\mathcal{E}$ is a locally free and $\mathbf{C}^{*}$-equivariant rank two coherent sheaf on $S$. The short exact sequence

$$
0 \rightarrow I_{1} \otimes L_{1} \rightarrow \mathcal{E} \rightarrow I_{2} \otimes L_{2} \rightarrow 0
$$

is then, by construction, also $\mathbf{C}^{*}$-equivariant. Since $\mathcal{E}$ is locally free, we must have $I_{1}=\mathcal{O}_{S}$.

Lemma 3.5. The support of $\mathcal{O} / I_{2}$ has empty intersection with $D$.

Proof. Because $\left.\mathcal{E}\right|_{D} \cong E$ by a $\mathbf{C}^{*}$-equivariant map, we may find, locally along $D$, some $\mathbf{C}^{*}$-invariant sections $s_{1}$ and $s_{2}$ of $\mathcal{E}$ that generate $\mathcal{E}$. These sections induce a $\mathbf{C}^{*}$-invariant isomorphism $\mathcal{E} \cong \mathcal{O}^{2}$. Thus, locally along $D$ we get a $\mathbf{C}^{*}$-invariant map $L_{1} \rightarrow \mathcal{O}^{2}$. Choosing a local $\mathbf{C}^{*}$-invariant generator of $L_{1}$ gives an expression of this map as $\psi: \mathcal{O} \rightarrow \mathcal{O}^{2}$, say with $\psi(s)=\left(f_{1} \cdot s, f_{2} \cdot s\right)$. Because $\psi$ is $\mathbf{C}^{*}$ equivariant, we find that both $f_{1}$ and $f_{2}$ are $\mathbf{C}^{*}$-invariant. But now if the image of $\left(f_{1}, f_{2}\right)$ vanishes in a fiber $\mathcal{E}_{p}$ of $\mathcal{E}$ at a point $p \in D$, then $\left(f_{1}, f_{2}\right)$ vanishes along $\pi^{-1}(\pi(p))$, hence $\mathcal{E} /\left(I_{1} \otimes L_{1}\right)$ has torsion supported along $\pi^{-1}(\pi(p))$. However, this quotient is torsion-free by construction, and we may conclude that $\left(f_{1}, f_{2}\right)$ fails to vanish at any point $p \in D$. Therefore $\mathcal{E} /\left(I_{1} \otimes L_{1}\right)$ is locally free at every point $p \in D$, completing the proof.

Next, assume, without loss of generality, that $\left.\mathcal{E}\right|_{f} \cong \mathcal{O}(l \sigma) \oplus \mathcal{O}((-1-l) \sigma)$ along a general fiber $f$, where $l \geq 0$. Write $L_{1}=\mathcal{O}_{S}(l \sigma) \otimes \pi^{*} B_{1}$ and $L_{2}=$ $\mathcal{O}_{S}((-1-l) \sigma) \otimes \pi^{*} B_{2}$.

Lemma 3.6. $I_{2}=\mathcal{O}_{S}$.

Proof. We have an exact sequence

$$
\begin{aligned}
\operatorname{Ext}^{1}\left(\left(\mathcal{O} / I_{2}\right) \otimes L_{2}, L_{1}\right) \mathbf{C}^{*} \rightarrow & \operatorname{Ext}^{1}\left(L_{2}, L_{1}\right)^{\mathbf{C}^{*}} \rightarrow \\
& \operatorname{Ext}^{1}\left(I_{2} \otimes L_{2}, L_{1}\right)^{\mathbf{C}^{*}} \rightarrow \operatorname{Ext}^{2}\left(\left(\mathcal{O} / I_{2}\right) \otimes L_{2}, L_{1}\right)^{\mathbf{C}^{*}} .
\end{aligned}
$$

Now $\operatorname{Ext}^{1}\left(\left(\mathcal{O} / I_{2}\right) \otimes L_{2}, L_{1}\right)=0$, since it is (after applying Serre duality) isomorphic to $H^{1}$ of a sheaf supported on a finite collection of points. Consequently, it is enough to show that $\operatorname{Ext}^{2}\left(\left(\mathcal{O} / I_{2}\right) \otimes L_{2}, L_{1}\right)^{\mathbf{C}^{*}}=0$ : then any equivariant extension pulls back from an extension of $L_{2}$ by $L_{1}$, which can only be locally free if already $L_{2}=I_{2} \otimes L_{2}$. So, we must compute $\operatorname{Ext}^{2}\left(\left(\mathcal{O} / I_{2}\right), L_{2}^{-1} \otimes L_{1}\right) \mathbf{C}^{*}$. Since

$$
L_{2}^{-1} \otimes L_{1}=\mathcal{O}((1+2 l) \sigma) \otimes \pi^{*}\left(B_{2}^{-1} \otimes B_{1}\right)
$$


and $\mathcal{O} / I_{2}$ has discrete support, we may replace $L_{2}^{-1} \otimes L_{1}$ by $\mathcal{O}((1+2 l) \sigma)$ for this computation. Now, locally along $\sigma$ the quotient $\mathcal{O} / I_{2}$ has generators of the form $z^{k_{1}}\left(l^{*} / s^{*}\right)^{k_{2}}$ with $z$ a uniformizer in the $\sigma$ direction and $l^{*} / s^{*}$ a parameter in the fiber direction. Here $\mathbf{C}^{*}$ acts by

$$
\lambda \cdot\left(z^{k_{1}}\left(l^{*} / s^{*}\right)^{k_{2}}\right)=\lambda^{-k_{2}} z^{k_{1}}\left(l^{*} / s^{*}\right)^{k_{2}}
$$

hence, using short exact sequences in the first variable of Ext, we may reduce to considering $\operatorname{Ext}^{2}\left(\mathbf{C}_{\chi}, \mathcal{O}((1+2 l) \sigma)\right)^{\mathbf{C}^{*}}$, where $\mathbf{C}^{*}$ acts on $\mathbf{C}_{\chi}$ with nonpositive weight $\chi$. If we resolve $\mathbf{C}_{\chi}$ locally by

$$
\mathcal{O}(-f-\sigma) \underset{\mathbf{C}}{\otimes} \mathbf{C}_{\chi} \rightarrow[\mathcal{O}(-f) \oplus \mathcal{O}(-\sigma)] \underset{\mathbf{C}}{\otimes} \mathbf{C}_{\chi} \rightarrow \mathcal{O} \underset{\mathbf{C}}{\otimes} \mathbf{C}_{\chi} \rightarrow \mathbf{C}_{\chi}
$$

we find that $\operatorname{Ext}^{2}\left(\mathbf{C}_{\chi}, \mathcal{O}((1+2 l) \sigma)\right)^{\mathbf{C}^{*}}$ is an invariant quotient of

$$
H^{0}\left(\mathcal{O}((2+2 l) \sigma) \otimes \mathcal{O}(f) \underset{\mathbf{C}}{\otimes} \mathbf{C}_{-\chi}\right)^{\mathbf{C}^{*}}
$$

But any $\mathbf{C}^{*}$-invariant section of $\mathcal{O}((2+2 l) \sigma) \underset{\mathbf{C}}{\otimes} \mathbf{C}_{-\chi}$ vanishes to order at least $2+2 l-\operatorname{deg} \chi$ along $\sigma$ and thus maps to zero in $\operatorname{Ext}^{2}\left(\mathbf{C}_{\chi}, \mathcal{O}((1+2 l) \sigma)\right)$.

Consequently, a $\mathbf{C}^{*}$-equivariant locally free sheaf is a $\mathbf{C}^{*}$-invariant extension of the form $0 \rightarrow L_{1} \rightarrow \mathcal{E} \rightarrow L_{2} \rightarrow 0$.

Lemma 3.7. The map

$$
\operatorname{Ext}^{1}\left(L_{2}, L_{1}\right)^{\mathbf{C}^{*}} \rightarrow \operatorname{Ext}^{1}\left(L_{2},\left.L_{1}\right|_{D}\right)^{\mathbf{C}^{*}}
$$

is an isomorphism.

Proof. We have

$$
\operatorname{Ext}^{1}\left(L_{2}, L_{1}\right)=H^{1}\left(S, L_{2}^{-1} \otimes L_{1}\right)=H^{1}\left(S, \mathcal{O}((1+2 l) \sigma) \otimes \pi^{*}\left(B_{2}^{-1} \otimes B_{1}\right)\right) .
$$

The Leray spectral sequence shows that this is just

$$
H^{1}\left(D, \pi_{*} \mathcal{O}((1+2 l) \sigma) \otimes B_{2}^{-1} \otimes B_{1}\right) ;
$$

furthermore, the restriction map is evidently just the map

$$
H^{1}\left(D, \pi_{*} \mathcal{O}((1+2 l) \sigma) \otimes B_{2}^{-1} \otimes B_{1}\right) \rightarrow H^{1}\left(D, \mathcal{O}_{D} \otimes B_{2}^{-1} \otimes B_{1}\right),
$$

which is given fiberwise by the map $H^{0}\left(\mathbf{P}^{1}, \mathcal{O}((1+2 l) \cdot p)\right) \rightarrow \mathbf{C}$ (where $p$ is the zero point in $\mathbf{P}^{1}$ ) arising from evaluation at infinity. Since the unique $\mathbf{C}^{*}$-invariant section of $\mathcal{O}_{\mathbf{P}^{1}}((1+2 l) \cdot p)$ under our normalization of the $\mathbf{C}^{*}$ action is the one that vanishes to order $1+2 l$ at $p$ (and hence is nonzero at infinity), the map $\left[\pi_{*} \mathcal{O}_{S}((1+2 l) \sigma)\right]^{\mathbf{C}^{*}} \rightarrow \mathcal{O}_{D}$ is an isomorphism, and the conclusion follows.

Finally, then, returning to the case in which $\mathcal{E}$ is not locally free, we see that $\mathcal{E}^{* *} / \mathcal{E}$ is a finite-length $\mathbf{C}^{*}$-invariant quotient of $\mathcal{E}^{* *}$. We may summarize as follows.

Proposition 3.8. Every $\mathbf{C}^{*}$-equivariant $\mathcal{E}$ is the kernel of a finite-length $\mathbf{C}^{*}$-equivariant quotient of a locally free $\mathbf{C}^{*}$-equivariant coherent sheaf $\mathcal{F}$. The sheaf $\mathcal{F}$ is uniquely determined by the sheaf $L_{1}$ in its canonical short exact sequence

$$
0 \rightarrow L_{1} \rightarrow \mathcal{F} \rightarrow L_{2} \rightarrow 0
$$

together with the inclusion $\left.L_{1}\right|_{D} \subset E$. 
Proof. Given $\mathcal{E}$, let $\mathcal{F}=\mathcal{E}^{* *}$. Then the extension in Equation 3.1 determines data of $L_{1}$ and the inclusion $\left.L_{1}\right|_{D} \subset E$, and Lemma 3.7 shows that this data in turn determines $\mathcal{F}$ as a framed bundle. Now the inclusion $\mathcal{E} \subseteq \mathcal{F}$ is $\mathbf{C}^{*}$-equivariant, hence the quotient $\mathcal{F} / \mathcal{E}$ is also $\mathbf{C}^{*}$-equivariant, as desired.

3.2.2. Fixed Points: The Equal Case. Suppose now that $\mathcal{E}$ is locally free of type $E$. Let

$$
0 \rightarrow\left(\pi^{*} E^{\prime}\right)(d \sigma) \rightarrow \mathcal{E} \rightarrow \mathcal{I}_{Z \subset Y}(d \sigma) \rightarrow 0
$$

denote the canonical exact sequence. The same argument as we used in Lemma 3.5 for the type $\mathrm{U}$ case shows that, if $\mathcal{E}$ is $\mathbf{C}^{*}$-invariant, then $\operatorname{supp} Z \subset \sigma$.

Consider the boundary map

$$
\operatorname{Ext}^{1}\left(\mathcal{I}_{Z \subset Y}(d \sigma),\left(\pi^{*} E^{\prime}\right)(d \sigma)\right) \rightarrow \operatorname{Ext}^{2}\left(\mathcal{O}_{Z}(d \sigma),\left(\pi^{*} E^{\prime}\right)(d \sigma)\right)
$$

coming from the short exact sequence in the first variable

$$
0 \rightarrow \mathcal{I}_{Z \subset Y} \rightarrow \mathcal{O}_{Y} \rightarrow \mathcal{O}_{Z} \rightarrow 0 .
$$

As in the case of type $\mathrm{U}$, any class in $\operatorname{Ext}^{1}\left(\mathcal{I}_{Z \subset Y}(d \sigma),\left(\pi^{*} E^{\prime}\right)(d \sigma)\right)$ whose image in $\operatorname{Ext}^{2}\left(\mathcal{O}_{Z}(d \sigma),\left(\pi^{*} E^{\prime}\right)(d \sigma)\right)$ is zero must define a 1-extension that is not locally free, unless $Z=\emptyset$. But

$$
\operatorname{Ext}^{2}\left(\mathcal{O}_{Z}(d \sigma),\left(\pi^{*} E^{\prime}\right)(d \sigma)\right)^{\mathbf{C}^{*}} \cong\left[\operatorname{Ext}^{2}\left(\mathcal{O}_{Z}, \mathcal{O}\right)^{2}\right]^{\mathbf{C}^{*}}=0
$$

because all weights in $\operatorname{Ext}^{2}\left(\mathcal{O}_{Z}, \mathcal{O}\right)$ are greater than or equal to one. So any $\mathbf{C}^{*}$ invariant 1-extension has singularities unless $Z=\emptyset$.

This analysis gives the following proposition.

Proposition 3.9. Every $\mathbf{C}^{*}$-invariant $E$-framed sheaf of type $E$ is the kernel of a $\mathbf{C}^{*}$-equivariant morphism $\left(\pi^{*} E\right)(d \sigma) \rightarrow \mathcal{Q}$ where $\mathcal{Q}$ is a finite-length $\mathbf{C}^{*}$-equivariant $\mathcal{O}_{S}$-module supported along $\sigma$.

\section{The Extension Lemma and its Relatives}

We first introduce some notation for this section. We fix a line bundle $B$ on a smooth complete curve $C$, and let $\mathcal{A}$ denote the quasicoherent $\mathcal{O}_{C}$-algebra $\mathcal{A}=$ $\operatorname{Sym}_{\mathcal{O}_{C}} B$.

We introduce also several variations on this notation: we let $I \subset \mathcal{A}$ denote the ideal $\oplus_{n \geq 1} \operatorname{Sym}_{\mathcal{O}_{C}}^{n} B, \widehat{\mathcal{A}}$ denote the completion of $\mathcal{A}$ with respect to $I$ (note that $\widehat{\mathcal{A}}$ is no longer a quasicoherent sheaf!), and $\mathcal{A}^{\circ}$ denote the localization of $\mathcal{A}$ with respect to $I$. If $T$ is a $\mathbf{C}$-scheme, we let $\widehat{\left(\mathcal{A}_{T}\right)}$ denote the completion of the pullback of $\mathcal{A}$ to $T$ and $\mathcal{A}_{T}^{\circ}$ denote the pullback of $\mathcal{A}^{\circ}$ to $T$. Most often in this context we will let $R$ denote a DVR with uniformizing element $\pi$ and field of fractions $K$, and then we will use either $\operatorname{Spec} R$ or $\operatorname{Spec} K$ as our scheme $T$; in these cases, we will abbreviate to $\mathcal{A}_{R}$, etc.

The following fundamental lemma, which is a simplified version of a result of Langton from [Lan75], is essential in establishing separatedness of some moduli stacks and "orbit completion" properties.

Extension Lemma. ([Nev00a]) Suppose $\mathcal{E}_{K}$ is a torsion-free coherent $\mathcal{A}$-module on $C_{K}$ of rank two, $\mathcal{E}_{R}^{\circ}$ is an $R$-flat family of torsion-free coherent $\mathcal{A}^{\circ}$-modules on $C_{R}$, and $\psi: \mathcal{E}_{K} \otimes_{\mathcal{A}_{K}} \mathcal{A}_{K}^{\circ} \longrightarrow \mathcal{E}_{R}^{\circ} \otimes_{\mathcal{A}_{R}^{\circ}} \mathcal{A}_{K}^{\circ}$ is an isomorphism of $\mathcal{A}^{\circ}$-modules on $C_{K}$. Then there is a unique $\mathcal{A}_{R}$-submodule $\mathcal{E}_{R} \subseteq \mathcal{E}_{K}$ of $\mathcal{E}_{K}$ for which 
1. $\left.\mathcal{E}_{R}\right|_{C_{K}}=\mathcal{E}_{K}$,

2. $\left.\left.\mathcal{E}_{R} \otimes_{\mathcal{A}_{R}} \mathcal{A}_{R}^{\circ}\right|_{\text {Spec } \mathcal{A}^{\circ}} \cong \mathcal{E}_{R}^{\circ}\right|_{\text {Spec } \mathcal{A}^{\circ}}$ compatibly with $\psi$, and

3. $\mathcal{E}_{R}$ is an $R$-flat family of torsion-free $\mathcal{A}$-modules on $C_{R}$.

The proof of this result uses the equivalence of the categories of quasicoherent $\mathcal{A}$-modules and quasicoherent sheaves on $\operatorname{Spec} \mathcal{A}$ to translate between commutative algebra and geometry.

The Extension Lemma has the following important corollary.

Corollary 4.1. ([Nev00a]) Let $S=\mathbf{P}(L \oplus \mathcal{O})$. Let $m: \mathbf{C}^{*} \times S \longrightarrow S$ denote the multiplication map given by $m(\lambda, s)=\lambda^{-1} \cdot s$. Fix a rank two torsion-free coherent sheaf $\mathcal{E}$ on $S$, with restriction to the divisor at infinity $\left.\mathcal{E}\right|_{D}=E$ a rank two vector bundle $E$ on $D$. Then $m^{*} \mathcal{E}$ extends to a rank two flat family of torsion-free coherent sheaves $\overline{\mathcal{E}}$ on $S$ parametrized by $\mathbf{C}$, so that

$$
\left.\overline{\mathcal{E}}\right|_{\mathbf{C} \times D} \cong E \otimes \mathcal{O}_{\mathbf{C}}
$$

We include the proof of this fact, because the proof arises again, in conjunction with the $\mathbf{C}^{*}$ action on $\mathcal{M}_{S}(E)$, in Section 6.3.

Proof. The map $m: \mathbf{C}^{*} \times S \longrightarrow S$ when restricted to $\mathbf{C}^{*} \times(S \backslash \sigma)$ extends to a map $\bar{m}: \mathbf{C} \times(S \backslash \sigma) \longrightarrow S \backslash \sigma$. One gets a sheaf $\tilde{\mathcal{E}}:=\bar{m}^{*} \mathcal{E}$, whose restriction to $\mathbf{C}^{*} \times(S \backslash \sigma)$ is canonically isomorphic to $m^{*} \mathcal{E}$. One clearly gets a framing $\tilde{\mathcal{E}} \stackrel{\bar{m}^{*} \phi}{\longrightarrow} E \otimes \mathcal{O}_{\mathbf{C}}$ from the framing $\mathcal{E} \stackrel{\phi}{\longrightarrow} E$. Restricting $\tilde{\mathcal{E}}$ to $\mathbf{C} \times(S \backslash(\sigma \cup D))$ and $\bar{m}^{*} \mathcal{E}$ to $\mathbf{C}^{*} \times(S \backslash D)$ gives exactly the input data for the Extension Lemma, and so determines a unique extension of $\tilde{\mathcal{E}}$ to $\mathbf{C} \times(S \backslash D)$. Finally Zariski gluing now pieces together $\overline{\mathcal{E}}$ from $\tilde{\mathcal{E}}$ and its extension across $\sigma$.

A slightly more conceptual restatement of this corollary is the following.

Corollary 4.2. Suppose $(\mathcal{E}, \phi)$ is an element of $\mathcal{M}_{S}(E)(\operatorname{Spec} \mathbf{C})$. Then a limit $\lim _{\lambda \rightarrow 0} \lambda \cdot(\mathcal{E}, \phi)$ exists in $\mathcal{M}_{S}(E)(\operatorname{Spec} \mathbf{C})$.

One has also a completeness property for the fixed-point set $\left(\mathcal{M}_{S}(E)\right)^{\mathbf{C}^{*}}$.

Proposition 4.3. ([Nev00a]) Suppose $R$ is a DVR, $K$ is its field of fractions, and $\left(\mathcal{E}_{K}, \phi_{K}\right) \in\left(\mathcal{M}_{S}(E)\right)^{\mathbf{C}^{*}}(\operatorname{Spec} K)$. Then $\left(\mathcal{E}_{K}, \phi_{K}\right)$ is the restriction of some family $\left(\mathcal{E}_{R}, \phi_{R}\right) \in\left(\mathcal{M}_{S}(E)\right)^{\mathbf{C}^{*}}(\operatorname{Spec} R)$.

We end this section with a tool that allows us to prove separatedness of the stack $\mathcal{M}_{S}(E)$ in some cases. We continue to use the notation of the earlier parts of this section without further comment.

Uniqueness Lemma. Suppose that $\mathcal{E}_{R}$ and $\mathcal{E}_{R}^{\prime}$ are $R$-flat families of torsion-free rank two $\mathcal{A}_{R}$-modules and that, for the associated families of torsion-free sheaves on $\mathrm{Spec} \mathrm{Sym}^{\bullet} B$, there is some neighborhood of $C_{R}$ in $\mathrm{Spec} \mathrm{Sym}^{\bullet} B$ on restriction to which the associated families of sheaves are locally free. Suppose furthermore that there are given isomorphisms

$$
\begin{aligned}
\mathcal{E}_{R} \otimes K & \rightarrow \mathcal{E}_{R}^{\prime} \otimes K \quad \text { and } \\
\mathcal{E}_{R} \otimes \widehat{\mathcal{A}}_{R} & \rightarrow \mathcal{E}_{R}^{\prime} \otimes \widehat{\mathcal{A}}_{R}
\end{aligned}
$$


that yield a commutative diagram

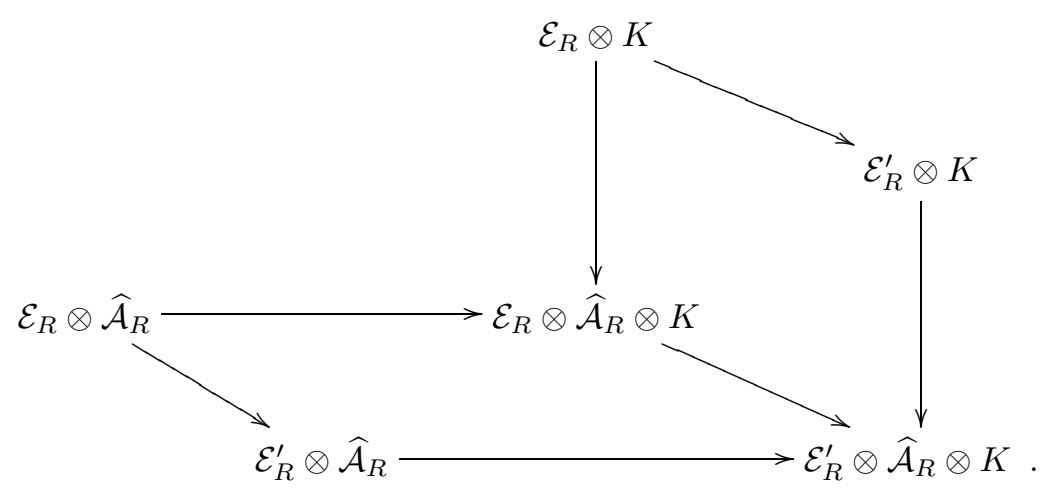

Then the given morphism $\mathcal{E}_{R} \otimes K \rightarrow \mathcal{E}_{R}^{\prime} \otimes K$ induces an isomorphism $\mathcal{E}_{R} \cong \mathcal{E}_{R}^{\prime}$ via the natural inclusions.

Proof. We will show that the images of $\mathcal{E}_{R}$ and $\mathcal{E}_{R}^{\prime}$ in $\mathcal{E}_{R} \otimes K$ and $\mathcal{E}_{R}^{\prime} \otimes K$, respectively, are identified under the given isomorphism.

Locally along $C$ we may trivialize $B$, and then over an open set $U_{R} \subset C_{R}$ we may replace $\mathcal{A}_{R}, \widehat{\mathcal{A}}_{R}$ by $T_{R}[t], T_{R}[[t]]$ respectively. Choose $f \in T_{R}[t] \backslash(t)$ so that $\left(\mathcal{E}_{R}\right)_{f}$ and $\left(\mathcal{E}_{R}^{\prime}\right)_{f}$ are free modules over $T_{R}[t]_{f}$; we may choose such an $f$ (assuming we have chosen $U_{R}$ sufficiently small) because $\mathcal{E}_{R}$ and $\mathcal{E}_{R}^{\prime}$ are locally free near $C_{R}$.

\section{Claim 4.4.}

a. $\left(\mathcal{E}_{R}\right)_{f}$ is the intersection of $\left(\mathcal{E}_{R}\right)_{f} \otimes K$ and $\left(\mathcal{E}_{R}\right)_{f} \underset{T_{R}[t]_{f}}{\otimes} T_{R}[[t]]$ in $\left(\mathcal{E}_{R}\right)_{f} \underset{T_{R}[t]_{f}}{\otimes} T_{R}[[t]] \underset{R}{\otimes} K$

b. $\left(\mathcal{E}_{R}^{\prime}\right)_{f}$ is the intersection of $\left(\mathcal{E}_{R}^{\prime}\right)_{f} \otimes K$ and $\left(\mathcal{E}_{R}^{\prime}\right)_{f} \underset{T_{R}[t]_{f}}{\otimes} T_{R}[[t]]$ in $\left(\mathcal{E}_{R}^{\prime}\right)_{f} \underset{T_{R}[t]_{f}}{\otimes} T_{R}[[t]] \underset{R}{\otimes} K$

Proof. By the choice of $f$ it is enough to show that

$$
T_{R}[t]_{f}=\left(T_{R}[t]_{f} \otimes K\right) \cap T_{R}[[t]]
$$

So, write an element of the intersection as

$$
\frac{g}{\pi^{j} f^{k}}=s, \text { where } g \in T_{R}[t] \text { and } s \in T_{R}[[t]] \text {. }
$$

Then $g=\pi^{j} f^{k} s \in \pi^{j} T_{R}[[t]]$. Therefore, all coefficients of $g$ are divisible by $\pi^{j}$, and $\frac{g}{\pi^{j}} \in T_{R}[t]$ after all; consequently, $\frac{g}{\pi^{j} f^{k}} \in T_{R}[t]_{f}$ as desired. This proves the claim. 
Claim 4.4, together with the commutative diagram (4.1), gives an isomorphism $\left(\mathcal{E}_{R}\right)_{f} \rightarrow\left(\mathcal{E}_{R}^{\prime}\right)_{f}$ so that the diagram

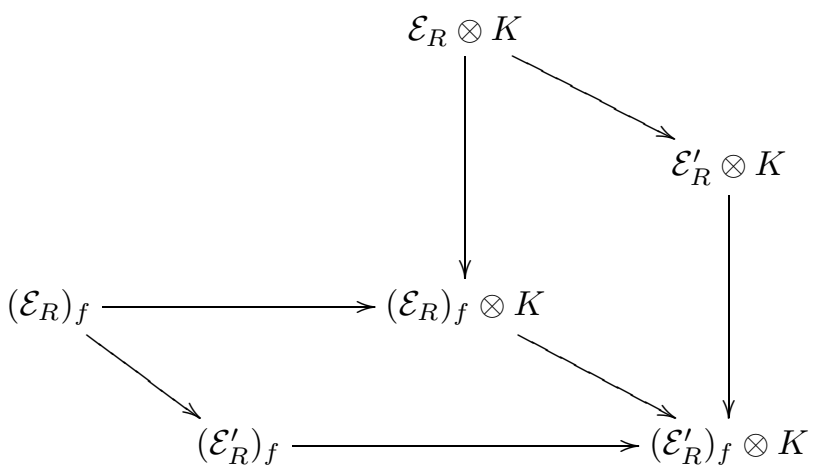

commutes. Then by Proposition 6 of [Lan75], the inclusions $\mathcal{E}_{R} \rightarrow \mathcal{E}_{R} \otimes K$ and $\mathcal{E}_{R}^{\prime} \rightarrow \mathcal{E}_{R}^{\prime} \otimes K$ induce an isomorphism of the $\mathcal{A}_{R}$-modules $\mathcal{E}_{R}$ and $\mathcal{E}_{R}^{\prime}$ over the open set $U_{R}$ of $C_{R}$. The isomorphisms so obtained locally along $C_{R}$ arise from the restrictions of a single diagram

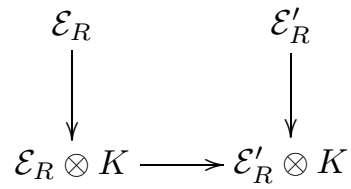

over $C_{R}$, so they are compatible on intersections of the open sets of the form $U_{R}$ and consequently induce an isomorphism $\mathcal{E}_{R} \rightarrow \mathcal{E}_{R}^{\prime}$ over all of $C_{R}$.

\section{Representability of the Stack}

Starting again in this section, we restrict to the case in which $C$ is an elliptic curve and $L$ has degree zero. Some related representability results may be found in [Leh93] and [Nev00b].

We begin by reviewing the description of polarizations of the surface $S \stackrel{\pi}{\longrightarrow} C$, since we will use this description below. First, recall that $\operatorname{Pic} S \cong \operatorname{Pic} C \oplus \mathbf{Z}$, where the factor $\mathbf{Z} \subset$ Pic $S$ may be generated by a section of the projection $\pi$. Suppose $\tau$ is a section of $\pi$ whose self-intersection $\tau^{2}$ is minimal among self-intersections of sections of $\pi$; note that $\tau^{2}$ is then the same as

$$
-\max \{2 \operatorname{deg} B-\operatorname{deg} L \mid B \text { is a subbundle of } L \oplus \mathcal{O}\} .
$$

If $\operatorname{deg} L \geq 0$, then this is just - $\operatorname{deg} L$, and the divisor at infinity $D$ is such a section. In this setting, one has (see [Fri98]) that $H=a D+b f$ (where $f$ is the numerical class of a fiber) is ample if and only if $a>0$ and $b>\frac{a}{2} \operatorname{deg} L$.

5.1. Elliptic Curves Equipped with Stable Bundles. It seems worth mentioning that it is not difficult to show that $\mathcal{M}_{S}(E)$ is a variety when $E$ is a stable bundle -indeed, one has the following.

Proposition 5.1. Suppose $C$ is a curve of any genus, $L$ has degree zero, $E$ is a rank two stable vector bundle on $D$, and $(\mathcal{E}, \phi) \in \mathcal{M}_{S}(E)(\operatorname{Spec} \mathbf{C})$. Then $\mathcal{E}$ is a stable sheaf (in the sense of Mumford-Takemoto) for an appropriate choice of polarization $H$ of $S$ that depends only on the Chern classes of $\mathcal{E}$. 
Proof. A straightforward computation shows that, when $a$ is sufficiently large (depending on $c_{1} \mathcal{E}$ and $c_{2} \mathcal{E}$ ) any such $\mathcal{E}$ will be stable for the polarization $H=$ $a D+b f$.

Proposition 5.1 has the following pleasant consequence.

Corollary 5.2. If $C$ is an elliptic curve, $E$ is stable, and $\operatorname{deg} L=0$, then $\mathcal{M}_{S}(E)$ is represented by a smooth quasiprojective variety.

Proof. This follows from the GIT construction and deformation theory of HuybrechtsLehn ([HL95b], [HL95a]).

5.2. Elliptic Curves Equipped with Polystable Bundles. By contrast, the case in which the bundle $E$ splits as $E=L_{1} \oplus L_{2}$ is not so straightforward, though the stack is still reasonably well behaved.

Theorem 5.3. Suppose that $C$ is an elliptic curve, that $L_{1}$ and $L_{2}$ are line bundles on $C$ both of the same arbitrary degree, and that $L$ is a line bundle of degree zero on $C$. Suppose further that $L, L_{1}$, and $L_{2}$ satisfy the very general conditions:

1. $L^{k}$ is nontrivial for all $k \in \mathbf{Z} \backslash\{0\}$; and

2. $L^{k} \otimes\left(L_{1}^{-1} \otimes L_{2}\right)$ is nontrivial for all $k \in \mathbf{Z}$.

Then if $S=\mathbf{P}(L \oplus \mathcal{O})$, the moduli stack $\mathcal{M}_{S}\left(L_{1} \oplus L_{2}\right)$ is a smooth separated scheme. Moreover, $\mathcal{M}_{S}\left(L_{1} \oplus L_{2}\right)$ is exhausted by open subschemes that are equivariantly quasiprojective with respect to the natural action of $\left(\mathbf{C}^{*}\right)^{3}$ on $\mathcal{M}_{S}\left(L_{1} \oplus L_{2}\right)$.

Proof. We may assume that $\operatorname{deg} L_{1}=\operatorname{deg} L_{2}=0$, since if we begin with $\operatorname{deg} L_{1}=$ $\operatorname{deg} L_{2}=d$ then for any point $p \in C, L_{1}^{\prime}=L_{1}(-d p)$ and $L_{2}^{\prime}=L_{2}(-d p)$ will satisfy the assumptions.

We begin by proving that, for any $\operatorname{Spec} \mathbf{C}$-valued point $(\mathcal{E}, \phi)$ of $\mathcal{M}_{S}\left(L_{1} \oplus L_{2}\right)$, we have $\operatorname{Ext}_{S}^{0}(\mathcal{E}, \mathcal{E}(-D))=\operatorname{Ext}_{S}^{2}(\mathcal{E}, \mathcal{E}(-D))=0$. In this computation, we will use repeatedly that $\operatorname{Ext}_{S}^{2}(\mathcal{E}, \mathcal{E}(-D))^{*}=\operatorname{Ext}_{S}^{0}(\mathcal{E}, \mathcal{E}(-\sigma))$.

Suppose first that, if $f$ denotes the generic fiber of the projection $\pi: S \rightarrow C$, we have $\left.\mathcal{E}\right|_{f} \cong \mathcal{O}(l) \oplus \mathcal{O}(l)$. Then any endomorphism of $\left.\mathcal{E}\right|_{f}$ that vanishes at infinity is zero; hence any $e \in \operatorname{Ext}_{S}^{0}(\mathcal{E}, \mathcal{E}(-D))$ is zero on the generic fiber of $\pi$ and consequently is zero on all of $S$. Similarly, $\operatorname{Ext}_{S}^{0}(\mathcal{E}, \mathcal{E}(-\sigma))=0$ and hence $\operatorname{Ext}_{S}^{2}(\mathcal{E}, \mathcal{E}(-D))=0$.

Suppose next that $\left.\mathcal{E}\right|_{f} \cong \mathcal{O}(l) \oplus \mathcal{O}\left(l^{\prime} \sigma\right)$ with $l>l^{\prime}$. Then, as in Section 3.1.1, we get a canonical exact sequence

$$
0 \rightarrow \pi^{*} B \otimes \mathcal{O}(l \sigma) \otimes I_{1} \rightarrow \mathcal{E} \rightarrow \pi^{*}\left(B^{-1} \otimes L_{1} \otimes L_{2}\right) \otimes \mathcal{O}\left(l^{\prime} \sigma\right) \otimes I_{2} \rightarrow 0
$$

Now

$$
\begin{aligned}
\operatorname{Ext}^{0}\left(\pi^{*}\left(B^{-1} \otimes L_{1} \otimes L_{2}\right) \otimes \mathcal{O}\right. & \left.\left(l^{\prime} \sigma\right) \otimes I_{2}, \pi^{*} B \otimes \mathcal{O}(l \sigma) \otimes I_{1}\right) \\
& \subseteq \operatorname{Ext}^{0}\left(\pi^{*}\left(B^{-1} \otimes L_{1} \otimes L_{2}\right) \otimes \mathcal{O}\left(l^{\prime} \sigma\right), \pi^{*} B \otimes \mathcal{O}(l \sigma)\right) \\
& =H^{0}\left(S, \pi^{*} B^{2} \otimes L_{1}^{-1} \otimes L_{2}^{-1} \otimes \mathcal{O}\left(\left(l-l^{\prime}\right) \sigma\right)\right) \\
& =H^{0}\left(C, B^{2} \otimes L_{1}^{-1} \otimes L_{2}^{-1} \otimes \sum_{j=0}^{l-l^{\prime}} L^{j}\right) .
\end{aligned}
$$


If $\operatorname{deg} B<0$, this group is zero, while if $\operatorname{deg} B=0$, then, since $L_{1} \oplus L_{2}$ is semistable of degree zero, we have either $B=L_{1}$ or $B=L_{2}$, and in either case the second assumption of the theorem implies that this group is zero.

Now if the canonical exact sequence is nonsplit, the above vanishing implies that $\mathcal{E}$ is simple, hence $\operatorname{Ext}_{S}^{0}(\mathcal{E}, \mathcal{E}(-D))=0$ as desired. If, on the other hand, the canonical exact sequence splits, then $B$ and $L_{1} \otimes L_{2} \otimes B^{-1}$ are line subbundles of the polystable bundle $L_{1} \oplus L_{2}$, and so $\operatorname{deg} B=0$ and either $B=L_{1}$ or $B=L_{2}$. This implies the $H^{0}$ vanishing just as before. To prove that $\operatorname{Ext}_{S}^{0}(\mathcal{E}, \mathcal{E}(-D))=0$ in this case, we must compute also

$$
\begin{aligned}
\operatorname{Ext}^{0}\left(\pi^{*} B \otimes \mathcal{O}(l \sigma) \otimes I_{1}, \pi^{*}\right. & \left.\left(B^{-1} \otimes L_{1} \otimes L_{2}\right) \otimes \mathcal{O}\left(l^{\prime} \sigma\right) \otimes I_{2}\right) \\
& \subseteq \operatorname{Ext}^{0}\left(\pi^{*} B \otimes \mathcal{O}(l \sigma), \pi^{*}\left(B^{-1} \otimes L_{1} \otimes L_{2}\right) \otimes \mathcal{O}\left(l^{\prime} \sigma\right)\right) \\
& =H^{0}\left(S, \pi^{*}\left(B^{-2} \otimes L_{1} \otimes L_{2}\right) \otimes \pi_{*} \mathcal{O}\left(\left(l^{\prime}-l\right) \sigma\right)\right) \\
& =0
\end{aligned}
$$

since $l^{\prime}<l$. Consequently,

$$
\begin{aligned}
\operatorname{Ext}^{0}(\mathcal{E}, \mathcal{E})= & \operatorname{End}\left(\pi^{*} B \otimes \mathcal{O}(l \sigma) \otimes I_{1}\right) \\
& \oplus \operatorname{End}\left(\pi^{*}\left(B^{-1} \otimes L_{1} \otimes L_{2}\right) \otimes \mathcal{O}\left(l^{\prime} \sigma\right) \otimes I_{2}\right)
\end{aligned}
$$

in this case. But the two sheaves in this formula are of rank one, hence $\operatorname{Ext}^{0}(\mathcal{E}, \mathcal{E})=$ $\mathbf{C}^{2}$ and the restriction map along $\sigma$ or along $D$ is just the identity of $\mathbf{C}^{2}$. As a result

$$
\operatorname{Ext}_{S}^{0}(\mathcal{E}, \mathcal{E}(-D))=\operatorname{Ext}_{S}^{2}(\mathcal{E}, \mathcal{E}(-D))=0
$$

here as well.

As a consequence of the vanishing of $\operatorname{Ext}_{S}^{0}(\mathcal{E}, \mathcal{E}(-D))$ for every Spec $\mathbf{C}$-point $(\mathcal{E}, \phi)$ of $\mathcal{M}_{S}\left(L_{1} \oplus L_{2}\right)$, we find that every object of $\mathcal{M}_{S}\left(L_{1} \oplus L_{2}\right)$ over Spec $\mathbf{C}$ is rigid. But then by semicontinuity every object of $\mathcal{M}_{S}\left(L_{1} \oplus L_{2}\right)$ is rigid as well, and by Propositions 4.4 and 1.4.1.1 of [LMB92], the stack $\mathcal{M}_{S}\left(L_{1} \oplus L_{2}\right)$ is in fact represented by a locally finitely presented algebraic space.

The deformation theory arguments used in [HL95a] to obtain a smoothness criterion for moduli spaces of stable framed sheaves are arguments purely about the smoothness of the moduli functor, and hence carry over mutatis mutandis to our setting; in particular, if $\operatorname{Ext}_{S}^{2}\left(\mathcal{E}, \mathcal{E} \stackrel{\phi}{\longrightarrow} L_{1} \oplus L_{2}\right)=0$ for all pairs $(\mathcal{E}, \phi)$, then $\mathcal{M}_{S}\left(L_{1} \oplus L_{2}\right)$ is smooth. But since $\phi$ induces an isomorphism $\left.\mathcal{E}\right|_{D} \cong L_{1} \oplus L_{2}$, we have that the complex $\mathcal{E} \stackrel{\phi}{\longrightarrow} L_{1} \oplus L_{2}$ is quasi-isomorphic to $\mathcal{E}(-D)$ concentrated in degree zero, and so

$$
\operatorname{Ext}_{S}^{2}\left(\mathcal{E}, \mathcal{E} \stackrel{\phi}{\longrightarrow} L_{1} \oplus L_{2}\right)=\operatorname{Ext}_{S}^{2}(\mathcal{E}, \mathcal{E}(-D))=0
$$

for all pairs $(\mathcal{E}, \phi)$. As a result, $\mathcal{M}_{S}\left(L_{1} \oplus L_{2}\right)$ is smooth.

Next, we need to prove that $\mathcal{M}_{S}\left(L_{1} \oplus L_{2}\right)$ is separated; so, writing $E=L_{1} \oplus L_{2}$, suppose that $\left(\mathcal{E}_{K}, \phi_{K}\right)$ is a family of $E$-framed torsion-free sheaves parametrized by Spec $K$, where $K$ is the field of fractions of a DVR $R$. We proceed in several steps.

Claim 5.4. Suppose $A$ is an affine scheme and $\left(\mathcal{E}_{A}, \phi_{A}\right)$ is an $E$-framed family parametrized by $A$. Then $\left.\mathcal{E}_{A}\right|_{\widehat{D}_{A}}$ is isomorphic to $\mathcal{O}_{\widehat{D}_{A}} \otimes_{\mathcal{O}_{D_{A}}}^{\otimes} E_{A}$; furthermore, this isomorphism is completely determined by $\phi_{A}$. 
Proof. Suppose we have shown that $\left.\mathcal{E}_{A}\right|_{D_{A}^{(n)}}$ is isomorphic to $\mathcal{O}_{D_{A}^{(n)}} \otimes E_{A}$. By Proposition 1.4 of [Gri66], the obstruction to uniqueness of an extension of $\mathcal{O}_{D_{A}^{(n)}} \otimes$ $E_{A}$ to a bundle over $D_{A}^{(n+1)}$ is a class in

$$
\begin{aligned}
& H^{1}\left(\text { End } E_{A} \otimes\left(I_{D}^{n} / I_{D}^{n+1}\right)\right) \cong H^{1}\left(\text { End } E \otimes\left(I_{D}^{n} / I_{D}^{n+1}\right)\right) \otimes A \\
&=\left[H^{1}\left(L^{n}\right) \oplus H^{1}\left(L^{n}\right) \oplus H^{1}\left(L_{1}^{-1} \otimes L_{2} \otimes L^{n}\right) \oplus H^{1}\left(L_{2}^{-1} \otimes L_{1} \otimes L^{n}\right)\right] \otimes A,
\end{aligned}
$$

which vanishes whenever $n \geq 1$ by the assumptions of the theorem. So $\left.\mathcal{E}_{A}\right|_{D_{A}^{(n+1)}} \cong$ $\mathcal{O}_{D_{A}^{(n+1)}} \otimes E_{A}$. Moreover, since

$$
\begin{array}{rlrl}
\operatorname{Hom}\left(E_{A}, E_{A} \otimes\left(I_{D}^{n} / I_{D}^{n+1}\right)\right) & =\left[H^{0}\left(L^{n}\right) \oplus H^{0}\left(L^{n}\right) \oplus H^{0}\left(L_{1}^{-1} \otimes L_{2} \otimes L^{n}\right)\right. \\
& =0 & \left.\oplus H^{0}\left(L_{2}^{-1} \otimes L_{1} \otimes L^{n}\right)\right] \otimes A
\end{array}
$$

whenever $n \geq 1$, we see that the isomorphism $\left.\mathcal{E}_{A}\right|_{D_{A}^{(n+1)}} \cong \mathcal{O}_{D_{A}^{(n+1)}} \otimes E_{A}$ is uniquely determined by $\phi_{A}$.

The same computation as in the proof of this claim gives

$$
\begin{aligned}
& \operatorname{End}\left(\mathcal{O}_{\widehat{D}_{R}} \otimes E_{R}\right) \cong \operatorname{End}\left(E_{R}\right) \cong \operatorname{End}(E) \otimes R, \quad \text { and } \\
& \operatorname{End}\left(K \otimes \mathcal{O}_{\widehat{D}_{R}} \otimes E_{K}\right) \cong \operatorname{End}\left(E_{K}\right) \cong \operatorname{End}(E) \otimes K .
\end{aligned}
$$

Suppose $\left(\mathcal{E}_{R}, \phi_{R}\right)$ and $\left(\mathcal{E}_{R}^{\prime}, \phi_{R}^{\prime}\right)$ are $E$-framed families parametrized by $\operatorname{Spec} R$ that are equipped with isomorphisms $\left.\left(\mathcal{E}_{R}, \phi_{R}\right)\right|_{S_{K}} \stackrel{\psi}{\longrightarrow}\left(\mathcal{E}_{K}, \phi_{K}\right)$ and $\left.\left(\mathcal{E}_{R}^{\prime}, \phi_{R}^{\prime}\right)\right|_{S_{K}} \stackrel{\psi^{\prime}}{\longrightarrow}$ $\left(\mathcal{E}_{K}, \phi_{K}\right)$. Restricting to $\widehat{D}_{R}$ and identifying each of $\left.\mathcal{E}_{R}\right|_{\widehat{D}_{R}},\left.\mathcal{E}_{R}^{\prime}\right|_{\widehat{D}_{R}}$ uniquely with $\mathcal{O}_{\widehat{D}_{R}} \otimes E_{R}$, we obtain an automorphism $a=\left.\left(\left(\psi^{\prime}\right)^{-1} \circ \psi\right)\right|_{\widehat{D}_{R} \otimes K}$ of $\left(K \otimes \mathcal{O}_{\widehat{D}_{R}}\right) \otimes E_{K}$ whose restriction to $E_{K}$ is the identity.

Claim 5.5. The automorphism $a$ is the restriction of the identity automorphism of $\mathcal{O}_{\widehat{D}_{R}} \otimes E_{R}$.

Proof. This follows from the description in Equations (5.1) and (5.2).

Now, restrict to $S_{R} \backslash \sigma_{R}$. We then have sheaves of $\mathcal{A}_{R}$-modules $\mathcal{E}_{R}$ and $\mathcal{E}_{R}^{\prime}$ where $\mathcal{A}=\operatorname{Sym}^{\bullet} L$, together with embeddings $\mathcal{E}_{R} \rightarrow \mathcal{O}_{\widehat{D}_{R}} \otimes \mathcal{E}_{R}$ and $\mathcal{E}_{R}^{\prime} \rightarrow \mathcal{O}_{\widehat{D}_{R}} \otimes \mathcal{E}_{R}^{\prime}$; moreover, the isomorphisms $\mathcal{O}_{\widehat{D}_{R}} \otimes \mathcal{E}_{R} \rightarrow \mathcal{O}_{\widehat{D}_{R}} \otimes E_{R}$ and $\mathcal{O}_{\widehat{D}_{R}} \otimes \mathcal{E}_{R}^{\prime} \rightarrow \mathcal{O}_{\widehat{D}_{R}} \otimes E_{R}$ identify $\mathcal{E}_{R}$ and $\mathcal{E}_{R}^{\prime}$ with subsheaves of $\mathcal{O}_{\widehat{D}_{R}} \otimes E_{R}$, and in addition the restrictions of their images to $D_{K}$ coincide. By the Uniqueness Lemma, then, the inclusions $\mathcal{E}_{R} \rightarrow \mathcal{E}_{K}$ and $\mathcal{E}_{R}^{\prime} \rightarrow \mathcal{E}_{K}$ have the same image and thus induce an isomorphism of $\mathcal{E}_{R}$ and $\mathcal{E}_{R}^{\prime}$ over $S_{R} \backslash \sigma_{R}$. Because this is compatible with the isomorphism given over $S_{K}$, by the Extension Lemma we obtain an isomorphism $\left(\mathcal{E}_{R}, \phi_{R}\right) \cong\left(\mathcal{E}_{R}^{\prime}, \phi_{R}^{\prime}\right)$. This completes the proof that $\mathcal{M}_{S}\left(L_{1} \oplus L_{2}\right)$ is separated.

We now indicate why $\mathcal{M}_{S}\left(L_{1} \oplus L_{2}\right)$ is in fact a scheme. Suppose $(\mathcal{E}, \phi)$ is a Spec $\mathbf{C}$-valued point of $\mathcal{M}_{S}\left(L_{1} \oplus L_{2}\right)$. Let $H$ denote a polarization of $S$, and, given an $\mathcal{O}_{S}$-module $\mathcal{F}$, write $\mathcal{F}(n):=\mathcal{F} \otimes H^{n}$. Choose $n$ sufficiently large that $\mathcal{E}(n)$ is globally generated and $H^{1}(\mathcal{E}(n))=H^{2}(\mathcal{E}(n))=0$; then the same will hold for all $\mathcal{F}$ occurring in pairs $(\mathcal{F}, \psi)$ that lie in an open neighborhood of $(\mathcal{E}, \phi)$ in $\mathcal{M}_{S}\left(L_{1} \oplus L_{2}\right)$. 
We saw above that $\left.\mathcal{E}\right|_{D^{(m)}} \cong \mathcal{O}_{D^{(m)}} \otimes E$ for any $m \geq 1$ and that the isomorphism is uniquely determined by $\phi$. For $m$ sufficiently large, this isomorphism determines an injection $H^{0}(\mathcal{E}(n)) \rightarrow H^{0}\left(\mathcal{O}_{D^{(m)}} \otimes E(n)\right)$; fix such an $m$.

We next construct a scheme representing a neighborhood of $(\mathcal{E}, \phi)$ in $\mathcal{M}_{S}\left(L_{1} \oplus L_{2}\right)$. There is a Grassmannian $G$ parametrizing subspaces of $H^{0}\left(\mathcal{O}_{D^{(m)}} \otimes E(n)\right)$ that are of dimension equal to the dimension of $H^{0}(\mathcal{E}(n))$. Over $S \times G$, in addition, there is a canonical subbundle $V$ of $\mathcal{O}_{S \times G} \otimes H^{0}\left(\mathcal{O}_{D^{(m)}} \otimes E(n)\right)$. Now the $\mathbf{C}^{*}$ action on $S$ induces an action on $D^{(m)}$, and thereby on the space $H^{0}\left(\mathcal{O}_{D^{(m)}} \otimes E(n)\right)$ and the Grassmannian $G$; furthermore the universal subbundle $V$ is $\mathbf{C}^{*}$-invariant when $\mathcal{O}_{S \times G} \otimes H^{0}\left(\mathcal{O}_{D^{(m)}} \otimes E(n)\right)$ is equipped with the $\mathbf{C}^{*}$-equivariant structure that incorporates both these actions. From this family $V$ on $S \times G / G$, one obtains a relative Quot-scheme $\mathcal{Q}$ together with a universal subsheaf $\mathcal{U}$ of $p_{S \times G}^{*} V$ on $S \times G \times \mathcal{Q}$ : this Quot-scheme parametrizes subsheaves of the bundles $V_{g}(g \in G)$ on $S$ with Hilbert polynomials coinciding with that of

$$
\operatorname{ker}\left(\mathcal{O}_{S} \otimes H^{0}(\mathcal{E}(n)) \rightarrow \mathcal{E}(n)\right)
$$

By construction $\mathbf{C}^{*}$ acts on the Quot-scheme $\mathcal{Q}$ as well. We have a surjective map

$$
\mathcal{O}_{S} \otimes H^{0}\left(\mathcal{O}_{D^{(m)}} \otimes E(n)\right) \rightarrow \mathcal{O}_{D^{(m)}} \otimes E(n),
$$

and there is a $\mathbf{C}^{*}$-invariant locally closed subscheme $\mathcal{Q}_{n}^{\prime}$ of $\mathcal{Q}$ parametrizing those $\mathcal{U}_{g \times q} \subset V_{g}$ on $S$ for which

1. $\operatorname{ker}\left(V_{g} \rightarrow E(n)\right)$ is contained in $\mathcal{U}_{g \times q}$,

2. $V_{g} / \mathcal{U}_{g \times q}$ is torsion-free, and

3. the induced map $\left.\left(V_{g} / \mathcal{U}_{g \times q}\right)\right|_{D} \rightarrow E(n)$ is an isomorphism.

Over $\mathcal{Q}_{n}^{\prime}$ we have a universal diagram

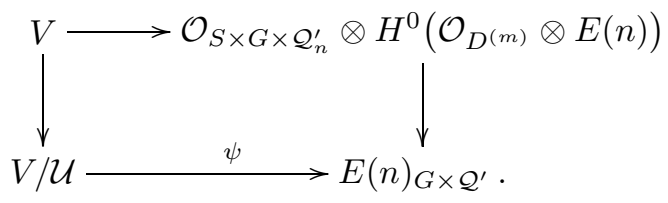

Claim 5.6. The map $\mathcal{Q}_{n}^{\prime} \rightarrow \mathcal{M}_{S}\left(L_{1} \oplus L_{2}\right)$ that is induced by the family

$\left((V / \mathcal{U})(-n),\left.\psi\right|_{D}(-n)\right) \mathbf{C}^{*}$-equivariantly represents a neighborhood of $(\mathcal{E}, \phi)$ in $\mathcal{M}_{S}\left(L_{1} \oplus L_{2}\right)$.

Proof. Suppose $\left(\mathcal{F}_{U}, \xi_{U}\right)$ is a $U$-flat family in $\mathcal{M}_{S}\left(L_{1} \oplus L_{2}\right)$ for which

1. the Hilbert polynomial of each $\mathcal{F}_{U, u}(u \in U)$ coincides with that of $\mathcal{E}$,

2. each $\mathcal{F}_{U, u}(n)$ is globally generated,

3. each $H^{1}\left(\mathcal{F}_{U, u}(n)\right)=H^{2}\left(\mathcal{F}_{U, u}(n)\right)=0$, and

4. each $H^{0}\left(\mathcal{F}_{U, u}(n)\right)$ injects into $H^{0}\left(\mathcal{O}_{D^{(m)}} \otimes E(n)\right)$ under the canonical homomorphism.

Then the family of diagrams

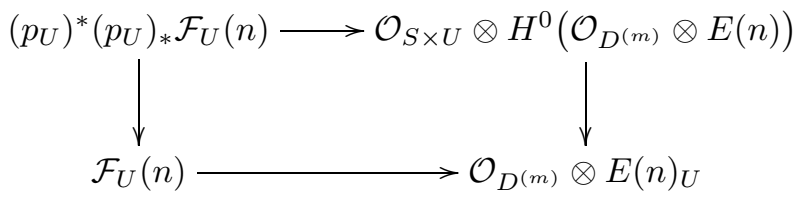


determines a map $U \rightarrow \mathcal{Q}_{n}^{\prime}$. This functor gives exactly the inverse of the map $\mathcal{Q}_{n}^{\prime} \rightarrow \mathcal{M}_{S}\left(L_{1} \oplus L_{2}\right)$, proving the claim.

This proves that $\mathcal{M}_{S}\left(L_{1} \oplus L_{2}\right)$ is exhausted by $\mathbf{C}^{*}$-invariant quasiprojective schemes, and so is itself a scheme.

In fact, $\mathcal{Q}_{n}^{\prime}$ is $\mathbf{C}^{*}$ equivariantly quasiprojective, and its image in $\mathcal{M}_{S}\left(L_{1} \oplus L_{2}\right)$ is $\mathbf{C}^{*}$-invariant: indeed, the usual construction of a relatively ample line bundle on a Quot-scheme $\mathcal{Q} \times G / G$ is given by first pushing forward to $\mathcal{Q} \times G$ a twist $(V / \mathcal{U})(\mu H)$ of the universal quotient $(V / \mathcal{U})$ on $S \times \mathcal{Q} \times G$ by a sufficiently high power $\mu$ of the pullback of an ample line bundle $\mathcal{O}_{S}(H)$ on $S$, and then taking the determinant bundle of this pushforward. Since this universal quotient is $\mathbf{C}^{*}$ equivariant, we obtain a $\mathbf{C}^{*}$-equivariant relatively ample line bundle on $\mathcal{Q} \times G / G$, hence, by restriction, on $\mathcal{Q}_{n}^{\prime} \times G / G$. As a consequence, we have in particular (see Section 4 of [BB73]) that $\mathcal{M}_{S}\left(L_{1} \oplus L_{2}\right)$ may be covered by $\mathbf{C}^{*}$-invariant quasi-affine open subschemes, and thus, by [BB73], $\mathcal{M}_{S}\left(L_{1} \oplus L_{2}\right)$ admits a Białynicki-Birula decomposition.

We will use this result in Section 6 when we compute a basis for the rational homology of $\mathcal{M}_{S}\left(L_{1} \oplus L_{2}\right)$.

\section{A Rational Homology Basis}

In this section, we use localization to compute a rational homology basis for the moduli space $\mathcal{M}_{S}\left(L_{1} \oplus L_{2}\right)$ in the case in which the line bundles $L, L_{1}$, and $L_{2}$ are of degree zero and satisfy the assumptions of Theorem 5.3.

6.1. The Localization Formula. The localization theory that we use was originally developed by Frankel ([Fra59]), Białynicki-Birula ([BB73], [BB74], [BB76]), and Carrell-Sommese ([CS79]); see also the work of Nakajima ([Nak99]), CarrellGoresky ([CG83]) and Kirwan ([Kir88]) for useful variations of the theory. The theorem that we want to use here is the following: if $M$ is a nonsingular algebraic variety with $\mathbf{C}^{*}$ action, one may compute the homology of $M$ in terms of that of the fixed-point set of the $\mathbf{C}^{*}$ action. More precisely, write $M^{\mathbf{C}^{*}}=\coprod_{\gamma} F_{\gamma}$, a disjoint union of connected components, and let

$$
S_{\gamma}=\left\{m \in M \mid \lim _{\lambda \rightarrow 0} \lambda \cdot m \in F_{\gamma}\right\} .
$$

Let $m_{\gamma}$ denote the complex codimension of $S_{\gamma}$ in $M$ (this is the same as the dimension of the negative weight space for the $\mathbf{C}^{*}$ action in a fiber of the normal bundle to $F_{\gamma}$ in $\left.M\right)$. Then

$$
H_{i}(M ; \mathbf{Q}) \cong \bigoplus_{\gamma} H_{i-2 m_{\gamma}}\left(F_{\gamma} ; \mathbf{Q}\right) .
$$

Originally this formula was proven assuming that $M$ is a projective variety or compact Kähler manifold. However, it has since been observed (see Chapter 5 of [Nak99]) that the same proof applies provided that $M$ is a smooth, Hausdorff Kähler manifold for which the flow lines $\left\{\lambda \cdot m \mid \lambda \in(0,1] \subset \mathbf{C}^{*}\right\}$ are contained in compact subsets of $M$ for all $m \in M$, using a Morse-theoretic decomposition of the manifold. In fact, the same proof also applies when one uses the (algebraically defined) Białynicki-Birula decomposition on a smooth separated scheme that is covered by $\mathbf{C}^{*}$-invariant quasiaffine open subschemes, provided a certain additional 
condition on the strata is satisfied; in Section 6.3, we describe this condition and prove that it is indeed satisfied by $\mathcal{M}_{S}\left(L_{1} \oplus L_{2}\right)$. This is the setting in which we apply the localization formula to compute rational homology.

6.2. Rational Homology of $\mathcal{M}_{S}\left(L_{1} \oplus L_{2}\right)$. We will use the same technique used by Nakajima in [Nak94a]: the bundle $L_{1} \oplus L_{2}$ has an action by $\mathbf{C}^{*} \times \mathbf{C}^{*}$ obtained by scaling in the factors, and this induces an additional $\mathbf{C}^{*} \times \mathbf{C}^{*}$ action on $\mathcal{M}_{S}\left(L_{1} \oplus L_{2}\right)$ by scaling the framing. By Proposition 4.3, the components of the fixed-point set are complete, and hence we may use localization for this action to reduce the computation to computation of the homology of the fixed-point set for the entire $\mathbf{C}^{*} \times \mathbf{C}^{*} \times \mathbf{C}^{*}$ action. Moreover, the coefficient giving the shift in homology grading, that is, the rank of the negative normal bundle to a component of the fixed-point set, will be the same whether one computes as though localization were iterated (that is, first localizing along the $\mathbf{C}^{*}$ fixed-point set and then localizing for the additional $\mathbf{C}^{*} \times \mathbf{C}^{*}$ action inside here) or performed for $\mathbf{C}^{*} \times \mathbf{C}^{*} \times \mathbf{C}^{*}$ all at once. Thus, we compute formally as though we were applying localization to the $\left(\mathbf{C}^{*}\right)^{3}$ action, although we will never consider any sort of completeness result for the entire $\left(\mathbf{C}^{*}\right)^{3}$ action.

The decomposition of rational homology that we will obtain is the following. Let $\mathcal{M}_{S}\left(L_{1} \oplus L_{2}\right)\left(c_{1}, c_{2}\right)$ denote the substack of $\mathcal{M}_{S}\left(L_{1} \oplus L_{2}\right)$ consisting of pairs $(\mathcal{E}, \phi)$ for which $c_{1} \mathcal{E}=c_{1}$ and $c_{2} \mathcal{E}=c_{2}$. Then

$$
\mathcal{M}_{S}\left(L_{1} \oplus L_{2}\right)=\coprod_{c_{1}, c_{2}} \mathcal{M}_{S}\left(L_{1} \oplus L_{2}\right)\left(c_{1}, c_{2}\right) .
$$

Theorem 6.1. Fixing $c_{1}=-l^{\prime} \sigma$ and $c_{2} \geq 0$, one has

$$
\begin{aligned}
H_{k}\left(\mathcal{M}_{S}\left(L_{1} \oplus L_{2}\right)\left(c_{1}, c_{2}\right) ; \mathbf{Q}\right) \cong \\
\bigoplus_{l \in \mathbf{Z}} \bigoplus_{\substack{\alpha, \beta \\
|\alpha|+|\beta|=c_{2}}} H_{k-2 d\left(\alpha, \beta, l, l^{\prime}\right)}\left(\operatorname{Sym}^{\alpha} C \times \operatorname{Sym}^{\beta} C ; \mathbf{Q}\right) .
\end{aligned}
$$

Here $d\left(\alpha, \beta, l, l^{\prime}\right)$, which depends on partitions

$$
\alpha=\left(1^{a_{1}} 2^{a_{2}} 3^{a_{3}} \ldots\right) \quad \text { and } \beta=\left(1^{b_{1}} 2^{b_{2}} 3^{b_{3}} \ldots\right),
$$

is given by

$$
\begin{array}{r}
d\left(\alpha, \beta, l, l^{\prime}\right)=\left[|\alpha|-\ell(\alpha)+\sum_{j>i \geq 0} a_{j}\left(1-\delta_{l^{\prime}+2 l-i-1,0}\right)\right] \\
+\left[|\beta|-\ell(\beta)+\sum_{j>i \geq 0} b_{j}\left(1-\delta_{l^{\prime}+2 l+i, 0}\right)\right],
\end{array}
$$

where $|\alpha|=\sum_{i \geq 1} i a_{i}$ and $\ell(\alpha)=\sum_{i \geq 1} a_{i}$.

6.3. Preliminaries. We begin by clarifying the nature of the fixed-point set and then confirming that the localization formula does indeed apply to $\mathcal{M}_{S}\left(L_{1} \oplus L_{2}\right)$.

Lemma 6.2. Let $E$ denote $L_{1} \oplus L_{2}$. Suppose $(\mathcal{E}, \phi) \in \mathcal{M}_{S}(E)(\operatorname{Spec} \mathbf{C})$ satisfies $m_{\lambda}^{*}(\mathcal{E}, \phi) \cong(\mathcal{E}, \phi)$ for all $\lambda \in \mathbf{C}^{*}$. Then $\mathcal{E}$ admits a $\mathbf{C}^{*}$-equivariant structure that makes $\phi \mathbf{C}^{*}$-equivariant for the trivial action on $L_{1} \oplus L_{2}$. 
Proof. Let $p: \mathbf{C}^{*} \times S \rightarrow S$ denote the projection to $S, q: \mathbf{C}^{*} \times S \rightarrow \mathbf{C}^{*}$ denote the projection to $\mathbf{C}^{*}$ and, by abuse of notation, also the projection $q: \mathbf{C}^{*} \times D \rightarrow \mathbf{C}^{*}$ to $\mathbf{C}^{*}$. We have $m_{\lambda}^{*} \mathcal{E} \cong \mathcal{E}$ for each $\lambda \in \mathbf{C}^{*}$ by assumption, and so $\operatorname{Ext}_{q}^{0}\left(m^{*} \mathcal{E}, p^{*} \mathcal{E}\right)$ is a vector bundle on $\mathbf{C}^{*}$ with fiber over $\lambda \in \mathbf{C}^{*}$ equal to $\operatorname{Hom}\left(m_{\lambda}^{*} \mathcal{E}, \mathcal{E}\right)$.

There is a natural restriction map

$$
\operatorname{Ext}_{q}^{0}\left(m^{*} \mathcal{E}, p^{*} \mathcal{E}\right) \rightarrow \operatorname{Ext}_{q}^{0}\left(\left.m^{*} \mathcal{E}\right|_{D},\left.p^{*} \mathcal{E}\right|_{D}\right)
$$

Now

$$
\begin{aligned}
\operatorname{Ext}_{q}^{0}\left(\left.m^{*} \mathcal{E}\right|_{D},\left.p^{*} \mathcal{E}\right|_{D}\right) & \cong \operatorname{Ext}_{q}^{0}\left(E_{\mathbf{C}^{*}}, E_{\mathbf{C}^{*}}\right) \\
& \cong \mathcal{O}_{\mathbf{C}^{*}} \otimes \operatorname{Hom}(E, E)
\end{aligned}
$$

via $\phi$, and, by assumption, the map

$$
\operatorname{Ext}_{q}^{0}\left(m^{*} \mathcal{E}, p^{*} \mathcal{E}\right) \rightarrow \mathcal{O}_{\mathbf{C}^{*}} \otimes \operatorname{Hom}(E, E)
$$

has in its image the identity element $1 \otimes \mathrm{id}$.

Furthermore, this map is injective: fiberwise we need only check the following.

Claim 6.3. $\operatorname{Hom}(\mathcal{E}, \mathcal{E}) \rightarrow \operatorname{Hom}\left(\left.\mathcal{E}\right|_{D},\left.\mathcal{E}\right|_{D}\right)$ is injective.

But this follows from the conjunction of

1. $\operatorname{Hom}(\mathcal{E}, \mathcal{E}) \rightarrow \operatorname{Hom}\left(\mathcal{E},\left.\mathcal{E}\right|_{D^{(n)}}\right)$ is injective for $n \gg 0$, since $\mathcal{E}$ is torsion-free; and

2. $\operatorname{Hom}\left(E, E \otimes\left(I_{D}^{n} / I_{D}^{n+1}\right)\right)=0$ for $n \geq 1$, and consequently

$$
\operatorname{Hom}\left(\mathcal{E},\left.\mathcal{E}\right|_{D^{(n)}}\right) \cong \operatorname{Hom}\left(\mathcal{E},\left.\mathcal{E}\right|_{D}\right) \text { for all } n \geq 1 \text {. }
$$

So the inverse image of $1 \otimes$ id in $\operatorname{Ext}_{q}^{0}\left(m^{*} \mathcal{E}, p^{*} \mathcal{E}\right)$ is uniquely determined. As in the proof of Lemma 3.3, uniqueness also guarantees commutativity of the diagram required for the map $m^{*} \mathcal{E} \rightarrow p^{*} \mathcal{E}$ to define a $\mathbf{C}^{*}$-equivariant structure.

Notation. We will continue to write $E$ in place of $L_{1} \oplus L_{2}$ and $\mathcal{M}_{S}(E)$ in place of $\mathcal{M}_{S}\left(L_{1} \oplus L_{2}\right)$ when it is convenient.

Lemma 6.4. Suppose $(\mathcal{E}, \phi) \in \mathcal{M}_{S}(E)(\operatorname{Spec} \mathbf{C})$ is invariant under the action of $\mathbf{C}^{*} \times \mathbf{C}^{*}$ by scaling in the factors $L_{1}$ and $L_{2}$. Then $\mathcal{E}$ splits as $\mathcal{E}=\mathcal{E}_{1} \oplus \mathcal{E}_{2}$ with $\left.\mathcal{E}_{1}\right|_{D} \cong L_{1}$ and $\left.\mathcal{E}_{2}\right|_{D} \cong L_{2}$ via $\phi$.

Proof. By Claim 6.3, the map $\operatorname{Hom}(\mathcal{E}, \mathcal{E}) \rightarrow \operatorname{Hom}\left(\mathcal{E},\left.\mathcal{E}\right|_{D}\right)$ is injective, so, because $\operatorname{End}\left(L_{1} \oplus L_{2}\right) \cong \mathbf{C}^{2}$ and the image of $\operatorname{Hom}(\mathcal{E}, \mathcal{E})$ in this group contains every $\left(\lambda_{1}, \lambda_{2}\right) \in \mathbf{C}^{*} \times \mathbf{C}^{*}$, we must have

$$
\operatorname{Hom}(\mathcal{E}, \mathcal{E}) \cong \operatorname{Hom}\left(\mathcal{E},\left.\mathcal{E}\right|_{D}\right)
$$

Consider the elements $e_{1}$ and $e_{2}$ in $\operatorname{Hom}(\mathcal{E}, \mathcal{E})$ that are the inverse images of the elements $(1,0)$ and $(0,1)$, respectively, of $\operatorname{End}\left(L_{1} \oplus L_{2}\right)$. The elements $e_{1}$ and $e_{2}$ are idempotents whose images are subsheaves (necessarily torsion-free) of $\mathcal{E}$; since their composites $e_{1} \circ e_{2}$ and $e_{2} \circ e_{1}$ are zero, the image of neither can have rank greater than 1 , but also $e_{1}+e_{2}=$ id and so $e_{1}(\mathcal{E}), e_{2}(\mathcal{E})$ must be rank one torsion-free subsheaves of $\mathcal{E}$ for which $\mathcal{E}=e_{1}(\mathcal{E})+e_{2}(\mathcal{E})$. Letting $\mathcal{E}_{i}=e_{i}(\mathcal{E})$ gives the desired splitting.

Suppose $(\mathcal{E}, \phi)$ is a fixed point for $\mathbf{C}^{*} \times \mathbf{C}^{*} \times \mathbf{C}^{*}$. Then $\mathcal{E}$ splits as

$$
\mathcal{E}=\left(\pi^{*} L_{1} \otimes \mathcal{O}(l \sigma) \otimes I_{1}\right) \bigoplus\left(\pi^{*} L_{2} \otimes \mathcal{O}\left(\left(-l^{\prime}-l\right) \sigma\right) \otimes I_{2}\right),
$$


where $I_{1}$ and $I_{2}$ are $\mathbf{C}^{*}$-invariant finite-colength ideals of $\mathcal{O}_{S}$.

Observe that $c_{1}(\mathcal{E})=-l^{\prime} \sigma$ and

$$
\begin{aligned}
c_{2}(\mathcal{E}) & =\left(\operatorname{deg} L_{1} \cdot f+l \sigma\right) \cdot\left(\operatorname{deg} L_{2} \cdot f-\left(l^{\prime}+l\right) \sigma\right)+c_{2} I_{1}+c_{2} I_{2} \\
& =c_{2} I_{1}+c_{2} I_{2} .
\end{aligned}
$$

Remark 6.5. In particular, neither $c_{1} \mathcal{E}$ nor $c_{2} \mathcal{E}$ depends on $l$, and hence even the part of $\mathcal{M}_{S}\left(L_{1} \oplus L_{2}\right)$ with fixed $c_{1}$ and $c_{2}$ may have rational homology groups of infinite rank (in fact, the formula of Theorem 6.1 makes it clear that it does). Note, however, because our ultimate goal is the computation of the action of Hecke operators, which will only move sheaves a bounded amount with respect to the invariant $l$, and since, as we will see, the homology basis we produce is finite in each value of the invariant $l$ (for fixed $c_{1}$ and $c_{2}$ ), the homology basis will still allow reasonable computations for Hecke operators.

We now describe the additional condition that $\mathcal{M}_{S}\left(L_{1} \oplus L_{2}\right)$ must satisfy in order for the localization formula to apply to it, and confirm that the condition is indeed satisfied.

In the Kähler case, the components $F_{\gamma}$ of the fixed-point set of $\mathbf{C}^{*}$ on the manifold $M$ are the critical submanifolds for a Morse-Bott function. This allows one to define a partial order on the index set $\{\gamma\}$ so that $\overline{S_{\gamma}} \subseteq \bigcup_{\delta \geq \gamma} S_{\delta}$; in particular, the partial order on the index set is the one generated by the relations: $\gamma \leq \delta$ if $\overline{S_{\gamma}} \cap S_{\delta} \neq \emptyset$. Atiyah and Bott ([AB83], pp. 536-537) describe how to use this "stratification with appropriate ordering" to obtain the localization formula.

Remark 6.6. The proof of the localization formula in the algebraic setting given by Białynicki-Birula does not require this partial ordering on the strata, but as his proof uses the Weil conjectures it is not so obvious to the author how one might try to modify his proof to apply to our noncompact spaces.

The crucial property for the partial ordering used by Atiyah-Bott is the following: suppose $\Gamma$ is a subset of the index set for the strata that satisfies

$$
\text { if } \gamma \in \Gamma \text { and } \mu \leq \gamma \text { then } \mu \in \Gamma \text {, }
$$

for which $\bigcup_{\gamma \in \Gamma} S_{\gamma}$ is the corresponding open submanifold of $M$. If $\delta$ is an index that is minimal among those indices that do not lie in $\Gamma$, then $S_{\delta}$ is a closed submanifold of $\left(\bigcup_{\gamma \in \Gamma} S_{\gamma}\right) \cup S_{\delta}$.

Our scheme $\mathcal{M}_{S}\left(L_{1} \oplus L_{2}\right)$ is the union of the increasing sequence $\left\{\mathcal{M}_{S}\left(L_{1} \oplus\right.\right.$ $\left.\left.L_{2}\right)_{m}\right\}$ of open subschemes that parametrize pairs $(\mathcal{E}, \phi)$ for which

$$
\left.\mathcal{E}\right|_{f} \cong \mathcal{O}(l) \oplus \mathcal{O}\left(-l^{\prime}-l\right)
$$

for some $l$ satisfying $\left(-l^{\prime} / 2\right) \leq l \leq m$ (here, as before, $f$ denotes the generic fiber of the projection $\pi: S \rightarrow C)$. We shall prove that if one of the Białynicki-Birula strata of $\mathcal{M}_{S}\left(L_{1} \oplus L_{2}\right)$ for the action of $\mathbf{C}^{*}$ has nonempty intersection with $\mathcal{M}_{S}\left(L_{1} \oplus L_{2}\right)_{m}$ then that stratum is contained in $\mathcal{M}_{S}\left(L_{1} \oplus L_{2}\right)_{m}$. We will next show that each $\mathcal{M}_{S}\left(L_{1} \oplus L_{2}\right)_{m}$ is quasiprojective (in particular, is Kähler) and consequently admits an ordering of its strata of the desired type; then, by semicontinuity of the "generic fiber type" $l$ in Equation (6.4), no stratum in $\mathcal{M}_{S}\left(L_{1} \oplus L_{2}\right)_{m+1} \backslash \mathcal{M}_{S}\left(L_{1} \oplus L_{2}\right)_{m}$ lies 
above any stratum in $\mathcal{M}_{S}\left(L_{1} \oplus L_{2}\right)_{m}$ in the ordering of strata of $\mathcal{M}_{S}\left(L_{1} \oplus L_{2}\right)_{m+1}$, and the localization argument applies to all $\mathcal{M}_{S}\left(L_{1} \oplus L_{2}\right)_{m}$.

We proceed, then, to show that $l$ is constant on strata of the decomposition for the action of $\mathbf{C}^{*}$ on $\mathcal{M}_{S}\left(L_{1} \oplus L_{2}\right)$.

\section{Proposition 6.7.}

1. Suppose that $(\mathcal{E}, \phi) \in \mathcal{M}_{S}\left(L_{1} \oplus L_{2}\right)(\operatorname{Spec} \mathbf{C})$, and that

$$
\left.\mathcal{E}\right|_{f} \cong \mathcal{O}(l) \oplus \mathcal{O}\left(-l^{\prime}-l\right) \text {. }
$$

Then, writing $\left(\mathcal{E}^{\prime}, \phi^{\prime}\right)=\lim _{\lambda \rightarrow 0} \lambda \cdot(\mathcal{E}, \phi)$, one also has

$$
\left.\mathcal{E}^{\prime}\right|_{f} \cong \mathcal{O}(l) \oplus \mathcal{O}\left(-l^{\prime}-l\right)
$$

2. If $(\mathcal{E}, \phi)$ and $\left(\mathcal{E}^{\prime}, \phi^{\prime}\right)$ lie in the same component of the fixed-point set of $\mathbf{C}^{*}$ in $\mathcal{M}_{S}\left(L_{1} \oplus L_{2}\right)$, then $\left.\left.\mathcal{E}\right|_{f} \cong \mathcal{E}^{\prime}\right|_{f}$.

Proof of (1). Suppose $U \subseteq C$ is an open set and $V=\pi^{-1}(U) \subseteq S$, for which

$$
\left.\left.\left.\mathcal{E}\right|_{V} \cong \mathcal{O}(l \sigma)\right|_{V} \oplus \mathcal{O}\left(\left(-l^{\prime}-l\right) \sigma\right)\right|_{V}
$$

Then by the construction of limits for orbits in Corollary 4.1, one has

$$
\begin{aligned}
\lim _{\lambda \rightarrow 0} \lambda \cdot\left(\left.\mathcal{E}\right|_{V}\right) & \left.\left.\cong \lim _{\lambda \rightarrow 0} \lambda \cdot \mathcal{O}(l \sigma)\right|_{V} \oplus \lim _{\lambda \rightarrow 0} \lambda \cdot \mathcal{O}\left(\left(-l^{\prime}-l\right) \sigma\right)\right|_{V} \\
& \left.\left.\cong \mathcal{O}(l \sigma)\right|_{V} \oplus \mathcal{O}\left(\left(-l^{\prime}-l\right) \sigma\right)\right|_{V}
\end{aligned}
$$

as desired.

Proof of (2). Since the components of the fixed-point set of $\mathbf{C}^{*}$ in $\mathcal{M}_{S}\left(L_{1} \oplus L_{2}\right)$ are smooth, the conclusion follows, provided any $\mathbf{C}^{*}$-equivariant deformation of $\mathcal{O}_{\mathbf{P}^{1}}(l)$

$\mathcal{O}_{\mathbf{P}^{1}}\left(-l^{\prime}-l\right)$ parametrized by an Artinian local $\mathbf{C}$-algebra is trivial; this, in turn, follows if

$$
\operatorname{Ext}_{\mathbf{P}^{1}}^{1}\left(\mathcal{O}(l) \oplus \mathcal{O}\left(-l^{\prime}-l\right), \mathcal{O}(l) \oplus \mathcal{O}\left(-l^{\prime}-l\right)\right)^{\mathbf{C}^{*}}=0
$$

This Ext ${ }^{1}$ reduces to $H^{1}\left(\mathbf{P}^{1}, \mathcal{O}\left(l^{\prime}+2 l\right) \oplus \mathcal{O}\left(-l^{\prime}-2 l\right)\right)^{\mathbf{C}^{*}}$; however the Čech complex computing $H^{*}\left(\mathbf{P}^{1}, \sum_{k} \mathcal{O}(k)\right)$ has no $\mathbf{C}^{*}$-invariants in $H^{1}$ : this is a consequence of the description in [Har77], Theorem III.5.1.

It remains just to show that each $\mathcal{M}_{S}\left(L_{1} \oplus L_{2}\right)_{m}$ is quasiprojective; but the method of proof used in Theorem 5.3 to show that $\mathcal{M}_{S}\left(L_{1} \oplus L_{2}\right)$ is a scheme will give the desired result, provided we can show that the sheaves $\mathcal{E}$ occurring in pairs $(\mathcal{E}, \phi)$ that lie in $\mathcal{M}_{S}\left(L_{1} \oplus L_{2}\right)_{m}$ (with fixed Chern classes $c_{1}$ and $c_{2}$ !) form a bounded family.

Suppose first that $\mathcal{E}$ is of type $U$. Then $\mathcal{E}$ occurs in the canonical exact sequence

$$
0 \rightarrow M_{1} \otimes I_{1} \rightarrow \mathcal{E} \rightarrow M_{2} \otimes I_{2} \rightarrow 0 .
$$

Write $M_{1}=\pi^{*} \mathcal{L} \otimes \mathcal{O}(l \sigma)$ and $\left.M_{2}=\pi^{*}(E / \mathcal{L}) \otimes \mathcal{O}\left(-l^{\prime}-l\right) \sigma\right)$ for some $l$ and some line subbundle $\mathcal{L} \subset E$ (recall that $E=L_{1} \oplus L_{2}$ ). Then

$$
c_{2} \mathcal{E}=-\left(l^{\prime}+2 l\right) \operatorname{deg} \mathcal{L}+c_{2} I_{1}+c_{2} I_{2} .
$$

One has $l \geq-l^{\prime} / 2$ and so

$$
-\left(l^{\prime}+2 l\right) \operatorname{deg} \mathcal{L} \geq 0 .
$$


Moreover, since $\mathcal{E}$ is of type $U$, one obtains $-\left(l^{\prime}+2 l\right)<0$, and consequently for each $l$ satisfying $\left(-l^{\prime} / 2\right)<l \leq m$ there are only finitely many possible values of $\operatorname{deg} \mathcal{L} \leq 0$ for which is it possible to satisfy Equation (6.5) with $c_{2} I_{1}, c_{2} I_{2} \geq 0$. But now for each of the finitely many possible collections of fixed data $l, \operatorname{deg} \mathcal{L}, c_{2} I_{1}$ and $c_{2} I_{2}$, such 1-extensions are parametrized by a subscheme of a projective bundle $\operatorname{over}_{\text {Quot }_{E / C}}(\operatorname{deg} \mathcal{L}) \times \operatorname{Hilb}_{S}^{c_{2} I_{1}} \times \operatorname{Hilb}_{S}^{c_{2} I_{2}}\left(\right.$ here Quot $_{E / C}(\operatorname{deg} \mathcal{L})$ denotes the Quotscheme parametrizing subsheaves of $E$ of degree $\operatorname{deg} \mathcal{L})$, thus giving boundedness.

Finally, suppose $\mathcal{E}$ is of type $E$. After twisting with $\mathcal{O}\left(l^{\prime} \sigma\right)$, we may assume $l^{\prime}=0$. If $\mathcal{E}$ is not locally free, we have $c_{2} \mathcal{E}=c_{2} \mathcal{E}^{* *}+\operatorname{length}\left(\mathcal{E}^{* *} / \mathcal{E}\right)$; moreover the formula of Section 4 of [Bro83a] shows that if $\mathcal{E}^{* *}$ has canonical exact sequence

$$
0 \rightarrow \mathcal{F}^{\prime} \rightarrow \mathcal{E}^{* *} \rightarrow \mathcal{I}_{Z \subset Y} \rightarrow 0
$$

then $c_{2} \mathcal{E}^{* *}=\operatorname{deg} Z$, so $c_{2} \mathcal{E}^{* *} \geq 0$ and there are only finitely many possible choices for $c_{2} \mathcal{E}^{* *}$ and length $\left(\mathcal{E}^{* *} / \mathcal{E}\right)$. Since a relative Quot-scheme for a bounded family (here we have in mind the Quot-scheme that parametrizes the quotients $\mathcal{E}^{* *} \rightarrow$ $\mathcal{E}^{* *} / \mathcal{E}$ of the vector bundle $\mathcal{E}^{* *}$ ) is itself a scheme of finite type, it will be enough to show that for each fixed choice of $c_{2} \mathcal{E}^{* *}$, the family of such vector bundles $\mathcal{E}^{* *}$ with the prescribed second Chern class is bounded.

Recall that $\mathcal{F}^{\prime}=\pi^{*} E^{\prime}$ for some rank two subsheaf $E^{\prime} \subseteq E$. Restricting the exact sequence (6.6) to $D$ gives

$$
\left.\left.\left.\mathcal{F}^{\prime}\right|_{D} \rightarrow \mathcal{E}^{* *}\right|_{D} \rightarrow \mathcal{I}_{Z \subset Y}\right|_{D} \rightarrow 0
$$

which must in fact be left exact as well (because $\left.\left.\mathcal{F}^{\prime}\right|_{D} \rightarrow \mathcal{E}^{* *}\right|_{D}$ is generically injective and $\left.\mathcal{F}^{\prime}\right|_{D}$ is torsion-free). There is a short exact sequence

$$
0 \rightarrow \mathcal{I}_{Z \subset Y} \rightarrow \mathcal{O}_{Y} \rightarrow \mathcal{O}_{Z} \rightarrow 0
$$

coming from the isomorphism $\mathcal{I}_{Z \subset Y} \cong \mathcal{I}_{Z} / \mathcal{I}_{Y}$, so the length of $\left.I_{Z \subset Y}\right|_{D}$ satisfies

$$
\begin{aligned}
\operatorname{length}\left(\left.\mathcal{I}_{Z \subset Y}\right|_{D}\right) & \leq \operatorname{length}\left(\left.\mathcal{O}_{Y}\right|_{D}\right)+\operatorname{length}\left(\operatorname{Tor}^{1}\left(\mathcal{O}_{D}, \mathcal{O}_{Z}\right)\right) \\
& \leq 2 \text { length }\left(\mathcal{O}_{Z}\right) .
\end{aligned}
$$

In particular,

$$
\operatorname{deg} E-2 \text { length }\left(\mathcal{O}_{Z}\right) \leq \operatorname{deg} E^{\prime} \leq \operatorname{deg} E .
$$

As a result, the collection of bundles $\mathcal{E}^{* *}$ may be parametrized by subschemes of projective bundles over finitely many products Quot $\times$ Hilb of a Quot-scheme for subsheaves of $E$ and a Hilbert scheme of points on $S$. This completes the proof of boundedness of the collection of sheaves $\mathcal{E}$ appearing in pairs $(\mathcal{E}, \phi) \in$ $\mathcal{M}_{S}(E)_{m}(\operatorname{Spec} \mathbf{C})=\mathcal{M}_{S}\left(L_{1} \oplus L_{2}\right)_{m}(\operatorname{Spec} \mathbf{C})$.

6.4. Proof of Theorem 6.1. The description of Equation 6.3 makes it clear what the components of the fixed-point set of $\mathcal{M}_{S}\left(L_{1} \oplus L_{2}\right)$ must be: they are isomorphic to products $\mathrm{Hilb}_{L}^{\alpha} \times \operatorname{Hilb}_{L}^{\beta}$ of $\mathbf{C}^{*}$-invariant sets of Hilbert schemes of points in the total space of $L$; these may be described (see [Nak99]) by partitions $\alpha, \beta$. We need therefore only compute the rank of the negative normal bundle along each component $\mathrm{Hilb}_{L}^{\alpha} \times \operatorname{Hilb}_{L}^{\beta}$. We may compute this rank at the general point of the component, that is, one for which $\operatorname{supp}\left(\mathcal{O} / I_{1}\right) \cap \operatorname{supp}\left(\mathcal{O} / I_{2}\right)=\emptyset$. 
Suppose $(\mathcal{E}, \phi)$ is such a general point; then the tangent space to $\mathcal{M}_{S}\left(L_{1} \oplus L_{2}\right)$ at $(\mathcal{E}, \phi)$ is $\operatorname{Ext}^{1}(\mathcal{E}, \mathcal{E}(-D))$. Now $\operatorname{Ext}^{1}(\mathcal{E}, \mathcal{E})$ splits into four components,

$$
\begin{aligned}
& \operatorname{Ext}^{1}\left(\pi^{*} L_{1} \otimes \mathcal{O}(l \sigma) \otimes I_{1}, \pi^{*} L_{1} \otimes \mathcal{O}(l \sigma) \otimes I_{1}\right), \\
& \operatorname{Ext}^{1}\left(\pi^{*} L_{2} \otimes \mathcal{O}\left(\left(-l^{\prime}-l\right) \sigma\right) \otimes I_{2}, \pi^{*} L_{2} \otimes \mathcal{O}\left(\left(-l^{\prime}-l\right) \sigma\right) \otimes I_{2}\right), \\
& \operatorname{Ext}^{1}\left(\pi^{*} L_{1} \otimes \mathcal{O}(l \sigma) \otimes I_{1}, \pi^{*} L_{2} \otimes \mathcal{O}\left(\left(-l^{\prime}-l\right) \sigma\right) \otimes I_{2}\right),
\end{aligned}
$$

and

$$
\operatorname{Ext}^{1}\left(\pi^{*} L_{2} \otimes \mathcal{O}\left(\left(-l^{\prime}-l\right) \sigma\right) \otimes I_{2}, \pi^{*} L_{1} \otimes \mathcal{O}(l \sigma) \otimes I_{1}\right)
$$

The first two factors are exactly the tangent spaces to the moduli spaces of rank one sheaves at $\pi^{*} L_{1} \otimes \mathcal{O}(l \sigma) \otimes I_{1}$ and $\pi^{*} L_{2} \otimes \mathcal{O}\left(\left(l^{\prime}-l\right) \sigma\right) \otimes I_{2}$, respectively, and the first-order deformations of these sheaves that vanish along $D$ are exactly the tangent spaces to the Hilbert schemes $T_{I_{1}} \operatorname{Hilb}_{L}^{\alpha}$ and $T_{I_{2}} \mathrm{Hilb}_{L}^{\beta}$, respectively. The negative normal bundles of these schemes have ranks $|\alpha|-\ell(\alpha)$ and $|\beta|-\ell(\beta)$, respectively (see Lemma 7.6 of [Nak99]).

It remains, then, to compute the negative weight spaces of the two remaining direct factors. We will use the short exact sequences

$$
0 \rightarrow \pi^{*} L_{1} \otimes \mathcal{O}(l \sigma) \otimes I_{1} \rightarrow \pi^{*} L_{1} \otimes \mathcal{O}(l \sigma) \rightarrow \pi^{*} L_{1} \otimes \mathcal{O}(l \sigma) \otimes \mathcal{O} / I_{1} \rightarrow 0
$$

and

$$
\begin{aligned}
0 \rightarrow \pi^{*} L_{2} \otimes \mathcal{O}\left(\left(-l^{\prime}-l\right) \sigma\right) \otimes I_{2} \rightarrow \pi^{*} L_{2} \otimes \mathcal{O}\left(\left(-l^{\prime}-l\right) \sigma\right) \rightarrow \\
\\
\pi^{*} L_{2} \otimes \mathcal{O}\left(\left(-l^{\prime}-l\right) \sigma\right) \otimes \mathcal{O} / I_{2} \rightarrow 0
\end{aligned}
$$

to induce long exact sequences in the first variable. Now

$$
\operatorname{Ext}^{1}\left(\pi^{*} L_{1} \otimes \mathcal{O}(l \sigma) \otimes \mathcal{O} / I_{1}, \pi^{*} L_{2} \otimes \mathcal{O}\left(\left(-l^{\prime}-l\right) \sigma\right) \otimes I_{2}\right)=0
$$

and

$$
\operatorname{Ext}^{1}\left(\pi^{*} L_{2} \otimes \mathcal{O}\left(\left(-l^{\prime}-l\right) \sigma\right) \otimes \mathcal{O} / I_{2}, \pi^{*} L_{1} \otimes \mathcal{O}(l \sigma) \otimes I_{1}\right)=0
$$

because the cosupports of $I_{1}$ and $I_{2}$ are disjoint. Similarly, the $\operatorname{Ext}^{2}$ term

$$
\operatorname{Ext}^{2}\left(\pi^{*} L_{1} \otimes \mathcal{O}(l \sigma), \pi^{*} L_{2} \otimes \mathcal{O}\left(\left(-l^{\prime}-l\right) \sigma\right) \otimes I_{2}\right)
$$

and its counterpart vanish because they sit in the exact sequence

$$
\begin{aligned}
& \operatorname{Ext}^{1}\left(\pi^{*} L_{1} \otimes \mathcal{O}(l \sigma), \pi^{*} L_{2} \otimes \mathcal{O}\left(\left(-l^{\prime}-l\right) \sigma\right) \otimes \mathcal{O} / I_{2}\right) \rightarrow \\
& \operatorname{Ext}^{2}\left(\pi^{*} L_{1} \otimes \mathcal{O}(l \sigma), \pi^{*} L_{2} \otimes \mathcal{O}\left(\left(-l^{\prime}-l\right) \sigma\right) \otimes I_{2}\right) \rightarrow \\
& \operatorname{Ext}^{2}\left(\pi^{*} L_{1} \otimes \mathcal{O}(l \sigma), \pi^{*} L_{2} \otimes \mathcal{O}\left(\left(-l^{\prime}-l\right) \sigma\right)\right)
\end{aligned}
$$

and its counterpart, respectively, in which the left and right terms vanish: the left vanishes because it computes $H^{1}$ of a skyscraper sheaf, the right because it computes

$$
H^{1}\left(C,\left(L_{1}^{-1} \otimes L_{2}\right)^{ \pm 1} \otimes \mathbf{R}^{1} \pi_{*} \mathcal{O}\left( \pm\left(l^{\prime}+2 l\right) \sigma\right)\right),
$$

which vanishes as in the proof of Theorem 5.3. 
Thus, to compute the remaining negative weight spaces of the tangent space to $\mathcal{M}_{S}\left(L_{1} \oplus L_{2}\right)$, it is enough to compute the negative weight spaces of

$$
\begin{aligned}
& \operatorname{Ext}^{1}\left(\pi^{*} L_{1} \otimes \mathcal{O}(l \sigma), \pi^{*} L_{2} \otimes \mathcal{O}\left(\left(-l^{\prime}-l\right) \sigma\right) \otimes I_{2}\right), \\
& \operatorname{Ext}^{1}\left(\pi^{*} L_{2} \otimes \mathcal{O}\left(\left(-l^{\prime}-l\right) \sigma\right), \pi^{*} L_{1} \otimes \mathcal{O}(l \sigma) \otimes I_{1}\right), \\
& \operatorname{Ext}^{2}\left(\pi^{*} L_{1} \otimes \mathcal{O}(l \sigma) \otimes \mathcal{O} / I_{1}, \pi^{*} L_{2} \otimes \mathcal{O}\left(\left(-l^{\prime}-l\right) \sigma\right)\right),
\end{aligned}
$$

and

$$
\operatorname{Ext}^{2}\left(\pi^{*} L_{2} \otimes \mathcal{O}\left(\left(-l^{\prime}-l\right) \sigma\right) \otimes \mathcal{O} / I_{2}, \pi^{*} L_{1} \otimes \mathcal{O}(l \sigma)\right)
$$

Note that we may (as we have done) omit the ideals in the second variables of the Ext ${ }^{2}$ groups here because of the assumption that the cosupports of $I_{1}$ and $I_{2}$ are disjoint.

We have exact sequences for the Ext ${ }^{1}$ groups of Equations 6.8 and 6.9 in the second variable, but this time the vanishing of cohomology groups involving terms $\left(L_{1}^{-1} \otimes L_{2}\right)^{ \pm 1} \otimes L^{j}$ implies that in fact

$$
\begin{aligned}
\operatorname{Ext}^{1}\left(\pi^{*} L_{1} \otimes \mathcal{O}(l \sigma), \pi^{*} L_{2} \otimes\right. & \left.\mathcal{O}\left(\left(-l^{\prime}-l\right) \sigma\right) \otimes I_{2}\right) \cong \\
& \operatorname{Ext}^{0}\left(\pi^{*} L_{1} \otimes \mathcal{O}(l \sigma), \pi^{*} L_{2} \otimes \mathcal{O}\left(\left(-l^{\prime}-l\right) \sigma\right) \otimes \mathcal{O} / I_{2}\right)
\end{aligned}
$$

and

$$
\begin{aligned}
\operatorname{Ext}^{1}\left(\pi^{*} L_{2} \otimes \mathcal{O}\left(\left(-l^{\prime}-l\right) \sigma\right), \pi^{*} L_{1} \otimes \mathcal{O}(l \sigma) \otimes I_{1}\right) \cong \\
\operatorname{Ext}^{0}\left(\pi^{*} L_{2} \otimes \mathcal{O}\left(\left(-l^{\prime}-l\right) \sigma\right), \pi^{*} L_{1} \otimes \mathcal{O}(l \sigma) \otimes \mathcal{O} / I_{1}\right) .
\end{aligned}
$$

We now choose a sequence of integers $\left(w_{1}, w_{2}, w_{3}\right) \in \mathbf{Z}^{3}$, satisfying $w_{3} \gg w_{2} \gg$ $w_{1}>0$, and adjust the weights of the $\left(\mathbf{C}^{*}\right)^{2} \times \mathbf{C}^{*}$ action on $\mathcal{M}_{S}\left(L_{1} \oplus L_{2}\right)$ accordingly by precomposing with the map $\left(\mathbf{C}^{*}\right)^{2} \times \mathbf{C}^{*} \rightarrow\left(\mathbf{C}^{*}\right)^{2} \times \mathbf{C}^{*}$ determined by these three weights.

We have reduced our computation to the computation of the negative weight spaces in the four groups

$$
\begin{aligned}
& H^{0}\left(\mathcal{O}\left(\left(-l^{\prime}-2 l\right) \sigma\right) \otimes \mathcal{O} / I_{2}\right) \underset{\mathbf{C}}{\otimes} \mathbf{C}\left(w_{2}-w_{1}\right), \\
& H^{0}\left(\mathcal{O}\left(\left(l^{\prime}+2 l\right) \sigma\right) \otimes \mathcal{O} / I_{1}\right) \underset{\mathbf{C}}{\otimes} \mathbf{C}\left(w_{1}-w_{2}\right), \\
& \operatorname{Ext}^{2}\left(\mathcal{O} / I_{1}, \mathcal{O}\left(\left(-l^{\prime}-2 l\right) \sigma\right)\right) \underset{\mathbf{C}}{\otimes} \mathbf{C}\left(w_{2}-w_{1}\right),
\end{aligned}
$$

and

$$
\operatorname{Ext}^{2}\left(\mathcal{O} / I_{2}, \mathcal{O}\left(\left(l^{\prime}+2 l\right) \sigma\right)\right) \underset{\mathbf{C}}{\otimes} \mathbf{C}\left(w_{1}-w_{2}\right)
$$

Here $\mathbf{C}(\chi)$ denotes the one-dimensional representation of $\mathbf{C}^{*}$ with weight $\chi$.

Moreover, we have

$$
\begin{aligned}
H^{0}\left(\mathcal{O}\left(\left(-l^{\prime}-2 l\right) \sigma\right) \otimes \mathcal{O} / I_{2}\right) \otimes \mathbf{C}\left(w_{2}-w_{1}\right) \cong \\
H^{0}\left(\mathcal{O} / I_{2}\right) \otimes \mathbf{C}\left(w_{2}-w_{1}-w_{3}\left(l^{\prime}+2 l\right)\right)
\end{aligned}
$$

and

$$
\begin{gathered}
H^{0}\left(\mathcal{O}\left(\left(l^{\prime}+2 l\right) \sigma\right) \otimes \mathcal{O} / I_{1}\right) \otimes \mathbf{C}\left(w_{1}-w_{2}\right) \cong \\
H^{0}\left(\mathcal{O} / I_{1}\right) \otimes \mathbf{C}\left(w_{1}-w_{2}+w_{3}\left(l^{\prime}+2 l\right)\right) .
\end{gathered}
$$


Now, as in Lemma 3.6, if we decompose $\mathcal{O} / I_{j}$ by weight, say $\mathcal{O} / I_{j}=\bigoplus_{n \geq 0} V\left(-n w_{3}\right)$, with filtered pieces $F\left(-n w_{3}\right)=\bigoplus_{m \geq n} V\left(-m w_{3}\right)$, then $\mathbf{C}^{*}$ acts in

$$
\operatorname{Ext}^{2}\left(V\left(-n w_{3}\right), \mathcal{O}\right):=\operatorname{Ext}^{2}\left(F\left(-n w_{3}\right) / F\left(-(n+1) w_{3}\right), \mathcal{O}\right)
$$

with weight $(n+1) w_{3}$ : for, using the resolution

$$
\mathcal{O}(-\sigma-f) \rightarrow \mathcal{O}(-f) \oplus \mathcal{O}(-\sigma) \rightarrow \mathcal{O} \rightarrow \mathbf{C}
$$

gives

$$
\operatorname{Ext}^{2}(\mathbf{C}, \mathcal{O})=\operatorname{coker}(\mathcal{O}(\sigma) \oplus \mathcal{O}(f) \rightarrow \mathcal{O}(\sigma+f))
$$

locally, and this latter group has local generator $z^{-1}\left(l^{*} / s^{*}\right)^{-1}$, where $z$ is a local parameter for the curve $C$ and $l^{*} / s^{*}$ is a local variable in the fiber direction; but on this generator $\mathbf{C}^{*}$ acts (under the inverse action!) with weight $w_{3}$.

We may summarize the weight space decompositions, then, as follows. Write the partitions $\alpha$ and $\beta$ as $\alpha=1^{a_{1}} 2^{a_{2}} 3^{a_{3}} \ldots$ and $\beta=1^{b_{1}} 2^{b_{2}} 3^{b_{3}} \ldots$ Then

$$
\begin{aligned}
& H^{0}\left(\mathcal{O} / I_{2}\right) \otimes \mathbf{C}\left(w_{2}-w_{1}-w_{3}\left(l^{\prime}+2 l\right)\right)=\bigoplus_{j \geq 0} \bigoplus_{i=0}^{j-1} \mathbf{C}^{b_{j}}\left(w_{2}-w_{1}-w_{3}\left(l^{\prime}+2 l+i\right)\right), \\
& H^{0}\left(\mathcal{O} / I_{1}\right) \otimes \mathbf{C}\left(w_{1}-w_{2}+w_{3}\left(l^{\prime}+2 l\right)\right)=\bigoplus_{j \geq 0}^{j-1} \bigoplus_{i=0}^{\mathbf{C}^{a_{j}}}\left(w_{1}-w_{2}+w_{3}\left(l^{\prime}+2 l-i\right)\right),
\end{aligned}
$$

$\operatorname{Ext}^{2}\left(\mathcal{O} / I_{1}, \mathcal{O}\right) \otimes \mathbf{C}\left(w_{2}-w_{1}-w_{3}\left(l^{\prime}+2 l\right)\right)=$

$$
\bigoplus_{j \geq 0} \bigoplus_{i=0}^{j-1} \mathbf{C}^{a_{j}}\left(w_{2}-w_{1}+w_{3}\left(i+1-\left(l^{\prime}+2 l\right)\right)\right),
$$

and

$$
\begin{aligned}
& \operatorname{Ext}^{2}\left(\mathcal{O} / I_{2}, \mathcal{O}\right) \otimes \mathbf{C}\left(w_{1}-w_{2}+w_{3}\left(l^{\prime}+2 l\right)\right)= \\
& \bigoplus_{j \geq 0}^{j-1} \bigoplus_{i=0}^{j} \mathbf{C}^{b_{j}}\left(w_{1}-w_{2}+w_{3}\left(i+1+\left(l^{\prime}+2 l\right)\right)\right) .
\end{aligned}
$$

Adding all terms, we get

$$
\begin{aligned}
\bigoplus_{j \geq 0} \bigoplus_{i=0}^{j-1}\left[\mathbf{C}^{b_{j}}\left(w_{2}-w_{1}-w_{3}\left(l^{\prime}+2 l+i\right)\right) \oplus \mathbf{C}^{b_{j}}\left(w_{1}-w_{2}+w_{3}\left(l^{\prime}+2 l+i+1\right)\right) \oplus\right. \\
\left.\quad \mathbf{C}^{a_{j}}\left(w_{2}-w_{1}+w_{3}\left(i+1-l^{\prime}-2 l\right)\right) \oplus \mathbf{C}^{a_{j}}\left(w_{1}-w_{2}+w_{3}\left(l^{\prime}+2 l-i\right)\right)\right] .
\end{aligned}
$$

Now, under our assumption that $w_{3} \gg w_{2} \gg w_{1}$, we find that

$$
\begin{aligned}
& w_{2}-w_{1}-w_{3}\left(l^{\prime}+2 l+i\right)<0 \quad \text { iff } \quad l^{\prime}+2 l+i \geq 1, \\
& w_{1}-w_{2}+w_{3}\left(l^{\prime}+2 l+i+1\right)<0 \quad \text { iff } \quad l^{\prime}+2 l+i \leq-1, \\
& w_{2}-w_{1}+w_{3}\left(-l^{\prime}-2 l+i+1\right)<0 \quad \text { iff } \quad l^{\prime}+2 l-i>1 \text {, }
\end{aligned}
$$

and

$$
w_{1}-w_{2}+w_{3}\left(l^{\prime}+2 l-i\right)<0 \quad \text { iff } \quad l^{\prime}+2 l-i \leq 0 .
$$


In other words, for each choice of $i$ and $j$, we obtain in the negative weight space a copy of

$$
\begin{array}{rll}
\mathbf{C}^{a_{j}} \oplus \mathbf{C}^{b_{j}} & \text { provided } & l^{\prime}+2 l \neq-i, i+1 \\
\mathbf{C}^{a_{j}} & \text { provided } & l^{\prime}+2 l=-i \\
\mathbf{C}^{b_{j}} & \text { provided } & l^{\prime}+2 l=i+1 .
\end{array}
$$

This may be summarized as follows: we obtain $\mathbf{C}^{d}$, where $d$ is given by

$$
d=\sum_{j>i \geq 0} b_{j}\left(1-\delta_{l^{\prime}+2 l+i, 0}\right)+\sum_{j>i \geq 0} a_{j}\left(1-\delta_{l^{\prime}+2 l-i-1,0}\right) .
$$

The total rank of the negative normal bundle (expressed in terms of the partitions $\alpha$ and $\beta$ and the degrees $l^{\prime}$ and $l$ ) is then

$$
\begin{aligned}
d\left(\alpha, \beta, l, l^{\prime}\right)=\left[|\alpha|-\ell(\alpha)+\sum_{j>i \geq 0} a_{j}\left(1-\delta_{l^{\prime}+2 l-i-1,0}\right)\right] & \\
+ & {\left[|\beta|-\ell(\beta)+\sum_{j>i \geq 0} b_{j}\left(1-\delta_{l^{\prime}+2 l+i, 0}\right)\right], }
\end{aligned}
$$

which gives the expression for Equation 6.2. This completes the proof of Theorem 6.1 .

The generating function given in the introduction follows from this theorem by a straightforward computation.

\section{REFERENCES}

[AB83] M. F. Atiyah and R. Bott. The Yang-Mills equations over Riemann surfaces. Philos. Trans. Roy. Soc. London Ser. A, 308(1505):523-615, 1983.

[Bar] Vladimir Baranovsky. Moduli of sheaves on surfaces and action of the oscillator algebra. arxiv: math.AG/9811092.

[BB73] A. Białynicki-Birula. Some theorems on actions of algebraic groups. Ann. of Math. (2), 98:480-497, 1973.

[BB74] A. Białynicki-Birula. On fixed points of torus actions on projective varieties. Bull. Acad. Polon. Sci. Sér. Sci. Math. Astronom. Phys., 22:1097-1101, 1974.

[BB76] A. Bialynicki-Birula. Some properties of the decompositions of algebraic varieties determined by actions of a torus. Bull. Acad. Polon. Sci. Sér. Sci. Math. Astronom. Phys., 24(9):667-674, 1976

[Bra91] Steven B. Bradlow. Special metrics and stability for holomorphic bundles with global sections. J. Differential Geom., 33(1):169-213, 1991.

[Bro83a] J. Eric Brosius. Rank-2 vector bundles on a ruled surface. I. Math. Ann., 265(2):155-168, 1983.

[Bro83b] J. Eric Brosius. Rank-2 vector bundles on a ruled surface. II. Math. Ann., 266(2):199$214,1983$.

[CG83] J. B. Carrell and R. M. Goresky. A decomposition theorem for the integral homology of a variety. Invent. Math., 73(3):367-381, 1983.

[CS79] James B. Carrell and Andrew John Sommese. Some topological aspects of $\mathbf{C}^{*}$ actions on compact Kaehler manifolds. Comment. Math. Helv., 54(4):567-582, 1979.

[Fra59] Theodore Frankel. Fixed points and torsion on Kähler manifolds. Ann. of Math. (2), 70:1-8, 1959

[Fri98] Robert Friedman. Algebraic surfaces and holomorphic vector bundles. Springer-Verlag, New York, 1998

[GKV95] Victor Ginzburg, Mikhail Kapranov, and Éric Vasserot. Langlands reciprocity for algebraic surfaces. Math. Res. Lett., 2(2):147-160, 1995.

[Gri66] Phillip A. Griffiths. The extension problem in complex analysis. II. Embeddings with positive normal bundle. Amer. J. Math., 88:366-446, 1966. 
[Gro96] I. Grojnowski. Instantons and affine algebras. I. The Hilbert scheme and vertex operators. Math. Res. Lett., 3(2):275-291, 1996.

[Har77] Robin Hartshorne. Algebraic geometry. Springer-Verlag, New York, 1977. Graduate Texts in Mathematics, No. 52.

[HL95a] D. Huybrechts and M. Lehn. Framed modules and their moduli. Internat. J. Math., 6(2):297-324, 1995.

[HL95b] D. Huybrechts and M. Lehn. Stable pairs on curves and surfaces. J. Algebraic Geom., 4(1):67-104, 1995.

[Kir88] Frances Kirwan. Intersection homology and torus actions. J. Amer. Math. Soc., 1(2):385-400, 1988.

[KKO00] Anton Kapustin, Alexander Kuznetsov, and Dmitri Orlov. Noncommutative instantons and twistor transform. IASSNS-HEP-00/14, 2000.

[KM99] Herbert Kurke and Andreas Matuschke. On the structure of moduli spaces of framed vector bundles on rational and ruled surfaces. In Algebraic geometry: Hirzebruch 70 (Warsaw, 1998), pages 239-271. Amer. Math. Soc., Providence, RI, 1999.

[KV00] M. Kapranov and E. Vasserot. Kleinian singularities, derived categories and Hall algebras. Math. Ann., 316(3):565-576, 2000.

[Lan75] Stacy G. Langton. Valuative criteria for families of vector bundles on algebraic varieties. Ann. of Math. (2), 101:88-110, 1975.

[Leh93] Manfred Lehn. Modulräume gerahmter Vektorbündel. Universität Bonn Mathematisches Institut, Bonn, 1993. Dissertation, Rheinische Friedrich-Wilhelms-Universität Bonn, Bonn, 1992.

[LMB92] G. Laumon and L. Moret-Bailly. Champs algebriques. Prépublications, Université de Paris-Sud, Orsay, France, 1992.

[Mat89] Hideyuki Matsumura. Commutative ring theory. Cambridge University Press, Cambridge, second edition, 1989. Translated from the Japanese by M. Reid.

[Nak94a] Hiraku Nakajima. Homology of moduli spaces of instantons on ALE spaces. I. J. Differential Geom., 40(1):105-127, 1994.

[Nak94b] Hiraku Nakajima. Instantons on ALE spaces, quiver varieties, and Kac-Moody algebras. Duke Math. J., 76(2):365-416, 1994.

[Nak97] Hiraku Nakajima. Heisenberg algebra and Hilbert schemes of points on projective surfaces. Ann. of Math. (2), 145(2):379-388, 1997.

[Nak98] Hiraku Nakajima. Quiver varieties and Kac-Moody algebras. Duke Math. J., 91(3):515$560,1998$.

[Nak99] Hiraku Nakajima. Lectures on Hilbert schemes of points on surfaces. American Mathematical Society, Providence, RI, 1999.

[Nev00a] Thomas A. Nevins. Localization and moduli stacks of framed sheaves on ruled surfaces. Preprint, August 2000.

[Nev00b] Thomas A. Nevins. Representability for some moduli stacks of framed sheaves. Submitted, July 2000.

[Tha94] Michael Thaddeus. Stable pairs, linear systems and the Verlinde formula. Invent. Math., 117(2):317-353, 1994.

[Vie95] Eckart Viehweg. Quasi-projective moduli for polarized manifolds. Springer-Verlag, Berlin, 1995.

Department of Mathematics, University of Michigan, 525 East University Avenue, ANN Arbor, MI 48109-1109 USA

E-mail address: nevins@umich.edu 